\title{
Decaying grid turbulence in a strongly stratified fluid
}

\author{
By OLIVIER PRAUD, ADAM M. FINCHAM $\dagger$ \\ AND JOEL SOMMERIA \\ Laboratoire des Ecoulements Géophysiques et Industriels (LEGI) CNRS-UJF-INPG, \\ Coriolis, BP53, 38041 Grenoble, cedex9, France
}

(Received 5 September 2002 and in revised form 15 June 2004)

Grid turbulence experiments have been carried out in a stably stratified fluid at moderately large Reynolds numbers (160 based on the Taylor microscale). A scanning particle image velocimetry technique is used to provide time-resolved velocity fields in a relatively large volume. For late times, in the low-Froude-number regime, the flow consists of quasi-horizontal motion in a sea of weak internal gravity waves. Here the dynamics of the flow is found to be independent of the ambient stratification. Fundamental differences with two-dimensional turbulence, due to the strong vertical shearing of horizontal velocity, are observed. In this regime, a self-similar scaling law for the energy decay and the length-scale evolution is observed. This behaviour reflects a process of adjustment of the eddy aspect ratio based on a balance between the horizontal advective motion which tends to vertically decorrelate the flow and the dissipation due to strong vertical shear. The characteristic vertical size of the eddies grows according to a diffusion law and is found to be independent of the turbulence generation. The organization of the flow into horizontal layers of eddies separated by intense shear leads to a strong anisotropy of the dissipation: this has been checked by direct measurement of the different tensorial components of the viscous dissipation.

\section{Introduction}

The dynamical evolution of freely evolving turbulence in the presence of stable stratification is of great importance to the understanding of atmospheric and oceanic turbulence. The influence of stable density stratification on initially homogeneous three-dimensional turbulence has been studied by a number of workers. In one of the first experiments Dickey \& Mellor (1980) dropped a biplane grid through a fluid at rest, and Britter et al. (1983) dragged a grid through a stratified fluid. They obtained interesting data concerning the decay rate of the vertical velocity. Britter et al. (1983) have shown that the main effect of the buoyancy forces is to suppress the vertical velocity component. Experiments were performed by Stillinger, Helland \& Van Atta (1983) and Itsweire, Helland \& Van Atta (1986) in a close loop channel, stratified with ten layers of salt water, and similar work was done later in a wind tunnel using stratified heated air by Lienhard \& Van Atta (1990) and Yoon \& Warhaft (1990). All these experiments have characterized the initial collapse of the three-dimensional turbulence, the damping effect of the density stratification on turbulence, and the statistical properties of the flow at early time.

$\dagger$ Address for correspondence: AME Department, USC, $854 \mathrm{~W}$ 36th Place, Los Angeles, CA 90089-1191, USA 
As well as experiments, a number of three-dimensional numerical studies of forced or decaying stratified homogeneous turbulence have been performed, within the Boussinesq approximation. Riley, Metcalfe \& Weissman (1981), Herring \& Metais (1989) and Metais \& Herring (1989) have examined the vertical structure and the distribution of energy between wave and vortical modes. In these works the collapse of the turbulence under the effect of stratification was found to affect both the dynamical properties of the flow and its structure. They observed a reduction of energy transfer and an inhibition of vertical scale growth.

After the collapse of the turbulence, the effect of stratification becomes dominant and the flow evolves in the low-Froude-number regime. It is this regime in which we are interested here. In this regime, vertical displacement is inhibited by stratification, leaving only two possible modes of motion: internal waves and vortices with horizontal motion. The flow is then characterized by a field of quasi-two-dimensional vortices undulating in a sea of internal gravity waves (see Riley et al. 1981). Laboratory experiments on this stage of stratified turbulence (see Hopfinger 1987; Fincham, Maxworthy \& Spedding 1996; Bonnier, Eiff \& Bonneton 2000; de Rooij, Linden \& Dalziel 1999) and numerical simulation (see Riley et al. 1981; Herring \& Metais 1989; Kimura \& Herring 1996) have revealed that these vortices look like 'pancakes' with a vertical extent thinner than the horizontal scale. A fine review of this strongly stratified limit can be found in Riley \& Lelong (2000). In such a layered flow, energy dissipation is associated with the strong vertical shear (see Riley et al. 1981; Herring \& Metais 1989; Metais \& Herring 1989; Fincham et al. 1996). This strong vertical shear is supposed to be responsible for the profound difference observed between twodimensional turbulence and the late stages of stratified turbulence. However, the mechanism of layering is still unclear, and the selection and the evolution of vertical length scale also remain an open question. Experiments where no vertical scale is initially imposed (see Park, Whitehead \& Gnanadeskian 1994; Fincham et al. 1996; Billant \& Chomaz 2000a) show that layers can spontaneously emerge from twodimensional horizontal flow.

In the present paper, we study this low-Froude-number regime at higher Reynolds numbers than in previous studies, thanks to the large size of the facility. This allows to get one step closer to the ideal inertial regimes typical of the ocean and the atmosphere. A Reynolds number of 160 is reached based on the vertical Taylor microscale (36000 based on grid mesh and velocity), while the Froude number is smaller than 0.6. Our parameters are compared in figure 1 with previous studies of grid-generated turbulence in a stratified fluid.

The main objectives of this work are to characterize the decay of stratified turbulence and to study the role of coherent structures in this regime. We will show the profound differences that exist between this quasi-horizontal turbulence and purely two-dimensional turbulence. The organization of the flow into staggered layers of quasi-horizontal motion leads to a strong anisotropy of the dissipation process that will be described and quantified.

The paper is organized as follows. The experimental set-up and procedure are presented in $\S 2$. A general description of the flow is proposed in $\S 3$, including the three-dimensional structure, the energy decay and the evolution of the horizontal and vertical length scales. The spectral analysis of the flow is presented in $\S 4$. In $\S 5$ we consider the contribution of the internal waves to the fluid motion by splitting the velocity field into its vortical and wave parts. Finally, anisotropy and dissipation processes are discussed and quantified in $\S 6$. 


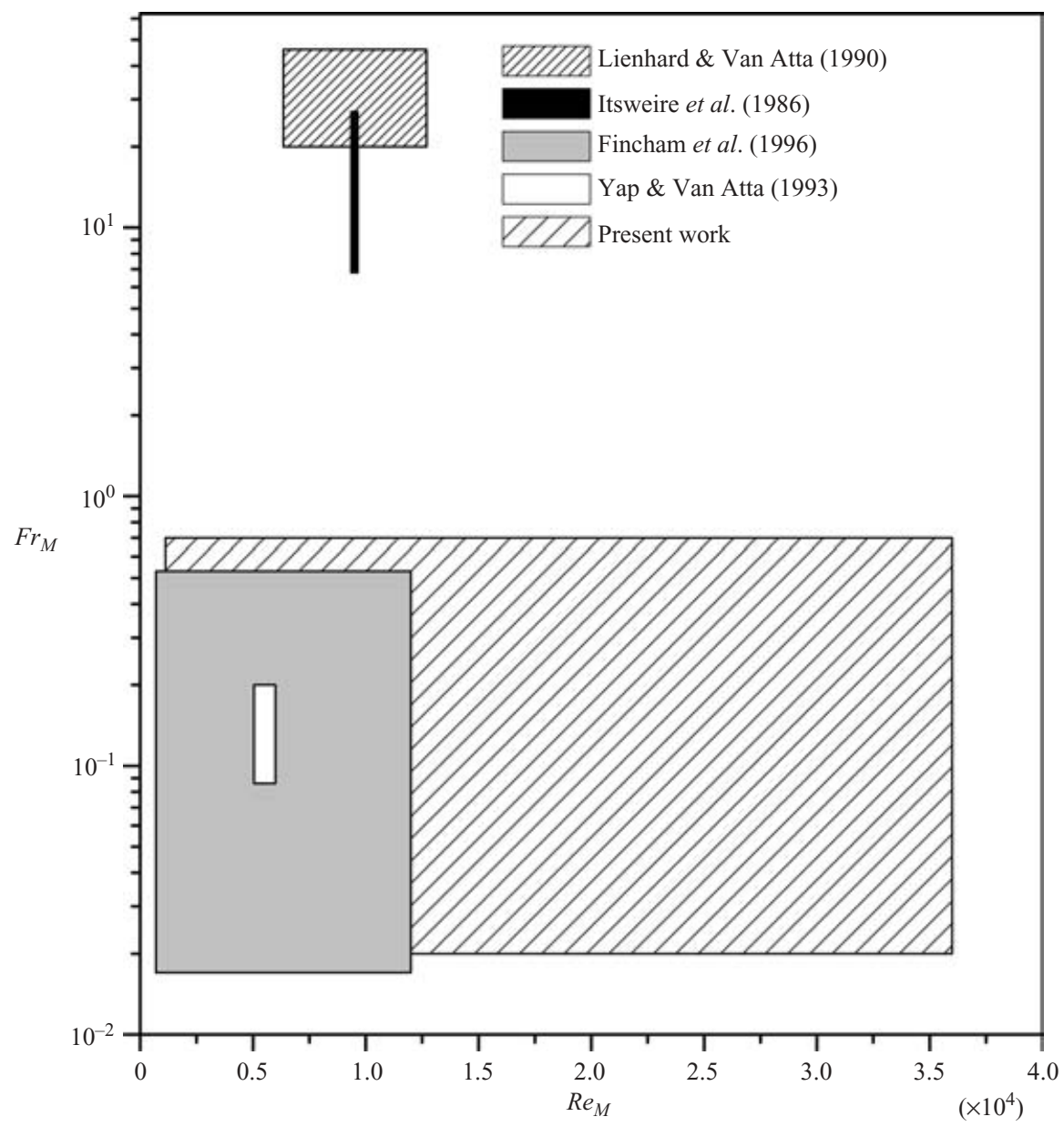

FiguRE 1. Range of Reynolds numbers, $R e_{M}$, and Froude numbers, $F r_{M}$, achieved in our experiments, compared with previous experiments on grid-generated turbulence $\left(\operatorname{Re}_{M}\right.$ and $\mathrm{Fr}_{M}$ are based on the grid mesh and velocity).

\section{Experimental set-up and procedure}

\subsection{Experimental apparatus}

The experiments were performed in a rectangular tank $(8 \mathrm{~m} \times 4 \mathrm{~m} \times 0.9 \mathrm{~m})$. It was linearly stratified using salt water. The salt stratification is established by means of computer-controlled volumetric pumps, from two tanks, one filled with salt water and the other one with pure water. A rake of vertical flat plates of width $W$ and mesh spacing $M$ (see figure 2) was towed at speed $U$ along the full length of the tank. Two rakes have been used, with $M=22.5$ and $45 \mathrm{~cm}$, and the corresponding plate width is 5 and $10 \mathrm{~cm}$ respectively. The rake was attached to a carriage above the free surface and its displacement was driven through a computer-controlled DC motor ensuring a constant velocity of translation $U$ and good repeatability of the experiments.

The velocity of the rake was increased linearly and at the end of the motion decreased to zero also linearly. Acceleration and deceleration were chosen to minimize the generation of internal waves produced by the local compression of the fluid 


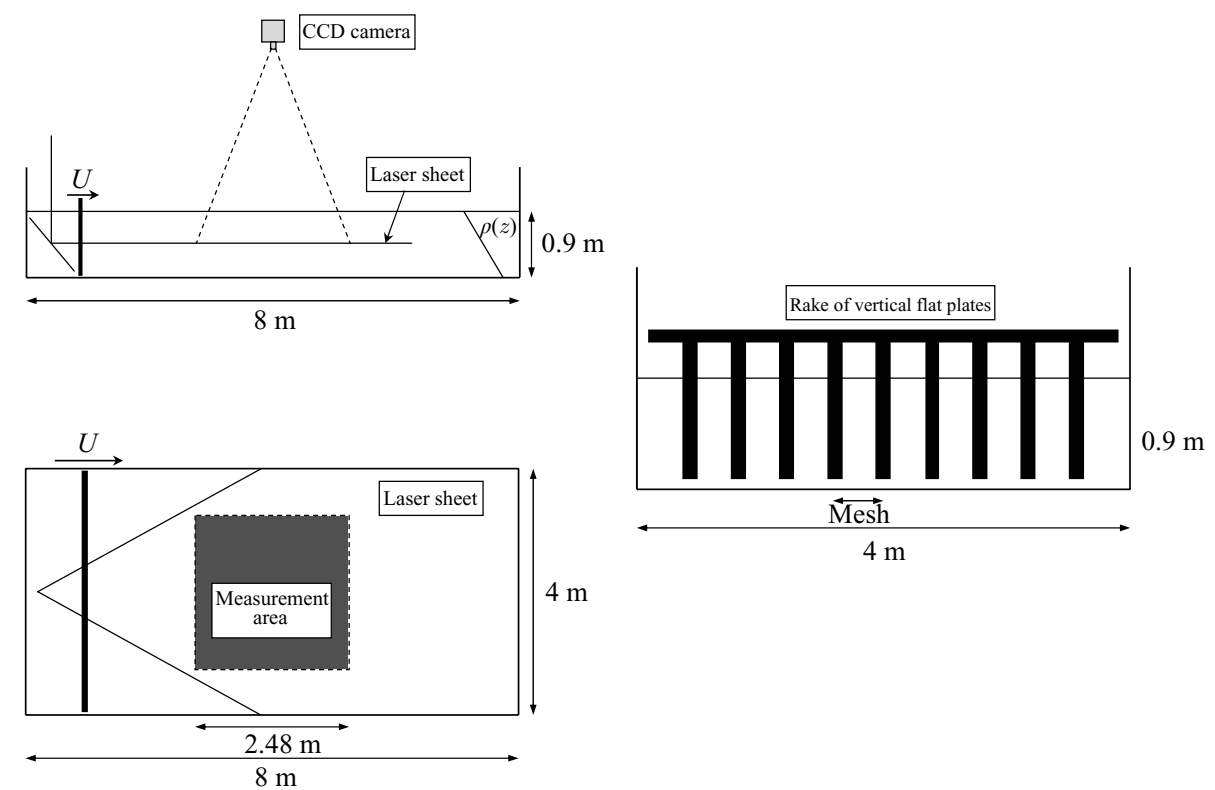

FIGURE 2. Side, top and front views of the experimental set-up.

between the rake and the edges of the closed tank. Plastic mesh baffles were placed at both ends of the channel to further damp any internal waves produced by the rake motion. The gap between the sidewalls and the grid was adjusted to be $M / 2$, as this was found to produce the smallest coherent backflow effect.

With the purpose of investigating the dynamics of stratified turbulence, the flow is forced two-dimensionally through vertical flat plates which minimizes both the internal wave generation and the mixing and does not select any particular vertical length scale. Moreover, plates were chosen instead of rods as they provide a welldefined separation point that is independent of the Reynolds number. By generating the turbulence by means of this rake, we introduce a maximum of kinetic energy in the horizontal modes (modes that result after the collapse of the three-dimensional turbulence in a stably stratified fluid) while avoiding large dissipation due to threedimensional turbulence that would be generated by a classical grid. Thus, even at the later stages of decay, the stratified turbulence is still energetic.

Stepper motors drove a profiling conductivity probe used to measure the density gradient in the tank and to verify the linearity of the stratification.

A high-resolution particle imaging velocimetry (PIV) system was used in these experiments to provide components and the spacial derivatives along the $x$-, $y$ - and $z$-directions of the horizontal velocity in a volume $(2.48 \mathrm{~m} \times 2.48 \mathrm{~m} \times 0.5 \mathrm{~m})$. The measurement volume is centred in the channel to minimize all the boundary effects. An origin of time $t_{0}$, based on the rake being located in the centre of the measurement area, is taken.

Figure 2 shows the experimental facility and the orientation of the measurement area.

\subsection{Particle imaging velocimetry measurement in a volume}

In these experiments, the correlation imaging velocimetry technique of Fincham \& Spedding (1997) was used to determine quantitatively the velocity field. More specifically the scanning correlation imaging velocimetry (SCIV) system of Fincham 
(1998) was used to explore its three-dimensional structure. The fluid was seeded with $600 \mu \mathrm{m}$ diameter polystyrene beads that were carefully prepared (by a process of cooking, which decreases slightly the density and successive density separations) to have a flat distribution of densities matching that of the salt stratification. This process ensures that there are equal number densities of particles at each depth. A photographic surfactant (Ilfotol) was added to the working fluid is small concentrations to prevent the polystyrene beads from agglomerating. The particle concentration must be sufficient to achieve good SCIV results but an excessive density leads to a serious damping of laser light. We use a typical concentration of 0.02 particle per pixel. The corresponding volume concentration is about $10^{-4} \mathrm{~g} \mathrm{~cm}^{-3}$ which does not perturb the flow properties. We can also derive an estimate of the characteristic time, $\tau_{s}$, needed for particles to attain velocity equilibrium with the fluid using Stockes drag: $\tau_{s}=d_{p}^{2} \rho_{p} / 18 \mu$, where $d_{p}$ is the diameter and $\rho_{p}$ the density of the tracer particles and $\mu$ the dynamic viscosity. Using $600 \mu \mathrm{m}$ diameter particles, the relaxation time is about $0.02 \mathrm{~s}$ which is much shorter than the characteristic time scale of the flow. We can therefore consider that the particles passively follow the fluid motion.

Coherent light originating from an $8 \mathrm{~W}$ argon laser was passed into an optical fibre the other end of which was attached to a small optical assembly which directs the light onto a small oscillating mirror (Cambridge Technology model 6850) creating a vertical light sheet. This small optical assembly moves horizontally on a linear bearing traverse. The light sheet then passes through a thin glass plate held parallel to and just touching the surface of the water above a $45^{\circ}$ mirror placed in the water. In this way, horizontal motion of the optical assembly above the water translates directly into vertical motion of the horizontal laser sheet within the fluid. Due to the refractive-index variation with depth, an initially horizontal beam of light will tend to curve downwards parabolically. In order to minimize this, only relatively weak stratifications were used and the angle of the horizontal light sheet was carefully inclined upwards a few degrees such that the summit of its parabolic trajectory corresponded to the centre of the measurement area. In this way the maximum deviation from horizontal occurs at the edges of the images and is limited to about $5 \mathrm{~mm}$ which is close to the light-sheet thickness.

The volume scanning process proceeded as follows: an initial scan through all depths is made with continuous image acquisition to memory, the light sheet is quickly returned to the starting position, and after an appropriate time interval the scan process is repeated to acquire the second image in each pair. The images are acquired by a $1024 \times 1024$ pixels 12 -bit SMD 1 M60 digital progressive scan camera operating at 60 f.p.s., placed $4 \mathrm{~m}$ above the free surface. Each slice (corresponding to a pair of images) is treated as described by Fincham \& Spedding (1997) and Fincham \& Delerce (2000). This correlation imaging velocimetry (CIV) technique is based on the calculation in physical space of the cross-correlation between local regions in the two images. The first image is divided into small rectangular boxes and the mean displacement of a given box is determined from the location of the maximum of the cross-correlation function. In an iterative way, the deformation of the fluid within each box is accounted for by locally deforming the images through use of a continuous function describing the discretely measured pixel intensities. As the true position of each velocity vector is located midway between the initial and final position of each pattern box, the velocity field is then reinterpolated onto a rectangular Cartesian grid using the thin-shell spline routines of Paihua (1978), as described in Spedding \& Rignot (1993). The resulting two-dimensional vector fields are combined into a cube that is fitted with a three-dimensional fourth-order tensor spline. Spatial derivatives 
along $x, y$ and $z$ are then computed analytically from the coefficients of the spline tensor. Typical measurement conditions produce $80 \times 80 \times 50$ independent vectors in a volume of $250 \times 250 \times 50 \mathrm{~cm}^{3}$. A combination of numerical simulations of the scanning technique, combined with actual hardware tests using real particles frozen inside a clear resin block, along with tests on stagnant fluid, showed that under optimum conditions the measured mean r.m.s. error in velocity is less than $2 \%$.

\subsection{Scales and parameters}

The relative importance of stratification is indicated by the Froude number, $F r=u / N L$, where $u$ is the r.m.s. turbulence velocity, $L$ a length scale of the energycontaining motion and $N=\sqrt{-g / \rho_{o}(\partial \rho / \partial z)}$ the buoyancy frequency, $\partial \rho / \partial z$ is the ambient density gradient and $\rho_{o}$ the mean density. The Froude number is a measure of the relative importance of the buoyancy effects compared to the inertial forces. In the early time after the generation of turbulence some patches with relatively large Froude number are found, suggesting the domination of the inertial effect. However, as the turbulence decays, the Froude number rapidly decreases as $u$ decreases and $L$ tends to grow. As time goes on, and turbulence decays further, $\mathrm{Fr}$ becomes small and the flow is completely dominated by stratification.

We define an initial Froude number, $F r_{M}$, from the mesh size $M$, the grid velocity $U$ and the Brunt-Väisälä frequency $N$, which are the main control parameters:

$$
F r_{M}=\frac{U}{N M} .
$$

We similarly define the initial Reynolds number $R e_{M}$ :

$$
R e_{M}=\frac{U M}{v}
$$

where $v$ is the mean kinematic viscosity, i.e. the viscosity of the salt solution at middepth of the tank (viscosity varies by $5.6 \%$ with depth for the highest stratification). For a given stratification, $\operatorname{Re}_{M}$ and $\mathrm{Fr}_{M}$ are related by $\operatorname{Re}_{M}=\mathrm{Fr}_{M} N M^{2} / v$ so that the Froude and Reynolds numbers vary together with $U$.

Experiments were performed for towing speeds $U$ of $0.5,1,2,4,8 \mathrm{~cm} \mathrm{~s}^{-1}$ and density gradients with buoyancy frequencies $N$ of 0.52 and $0.26 \mathrm{rad} \mathrm{s}^{-1}$. Two different grids were used: $M=45 \mathrm{~cm}$ and $M=22.5 \mathrm{~cm}$. The corresponding Reynolds and Froude numbers are in the range $1125<R e_{M}<36000$ and $0.02<F r_{M}<0.7$. Table 1 summarizes the parameters of the different experiments. The Reynolds number based on the vertical Taylor microscale, $R e_{\lambda}=u^{*} \lambda_{v}^{*} R e_{M}=0.85 R e_{M}^{1 / 2}$, is also indicated. Here the non-dimensional turbulent velocity $u^{*}$ and scale $\lambda_{v}^{*}$ are given from the measurements respectively using (3.1) and (3.6). This Reynolds number slightly decreases with time, and the data of table 1 are obtained with $t^{*} \equiv t U / M=10$, corresponding roughly to the beginning of the well-established turbulent decay.

\section{General description}

\subsection{Global decay of turbulence}

Depending on the experimental parameters, two different initial behaviours are observed. For low towing speeds, a laminar initial wake in which the motion remains two-dimensional and the vortices are initially columnar is observed. For higher towing speeds, the flow behind the rake is initially fully turbulent but rapidly collapses under the effect of stratification. 


\begin{tabular}{rcccccr}
\hline Exp & $U\left(\mathrm{~cm} \mathrm{~s}^{-1}\right)$ & $M(\mathrm{~cm})$ & $N\left(\mathrm{rad} \mathrm{s}^{-1}\right)$ & $\operatorname{Re}_{M}$ & $F_{M}$ & $\operatorname{Re}_{\lambda}$ \\
ba & 0.5 & 45 & 0.52 & 2250 & 0.021 & 40 \\
bb & 1 & 45 & 0.52 & 4500 & 0.043 & 57 \\
bc & 2 & 45 & 0.52 & 9000 & 0.086 & 81 \\
bd & 4 & 45 & 0.52 & 18000 & 0.17 & 114 \\
be & 8 & 45 & 0.52 & 36000 & 0.34 & 161 \\
aa & 0.5 & 22.5 & 0.52 & 1125 & 0.044 & 28 \\
ab & 1 & 22.5 & 0.52 & 2250 & 0.09 & 40 \\
ac & 2 & 22.5 & 0.52 & 4500 & 0.18 & 57 \\
ad & 4 & 22.5 & 0.52 & 9000 & 0.36 & 81 \\
ae & 8 & 22.5 & 0.52 & 18000 & 0.7 & 114 \\
ia & 0.5 & 45 & 0.26 & 2250 & 0.043 & 40 \\
ib & 1 & 45 & 0.26 & 4500 & 0.086 & 57 \\
ic & 2 & 45 & 0.26 & 9000 & 0.17 & 81 \\
id & 4 & 45 & 0.26 & 18000 & 0.34 & 114 \\
ie & 8 & 45 & 0.26 & 36000 & 0.7 & 161
\end{tabular}

TABLE 1. Parameters for the different experiments performed.

In both cases the final stage consists of quasi-horizontal motion strongly varying along the vertical. This flow organizes itself into interacting vortices. By pairing and merging, the typical size of the vortical structures grows until the large vortices scale like the width of the tank. The photographs (figure 3) show this process of energy transfer to larger scales and the formation of dominant vortical structures under the influence of the density stratification, as already noted by Lin \& Pao (1979) and Yap \& Van Atta (1993).

This evolution closely resembles two-dimensional turbulence in decoupled horizontal layers. The flow is quasi-horizontal, and the typical size of the structures increases with time. However, the strong vertical shearing between vortices in adjacent layers gives the flow a highly dissipative nature. Figure 4 shows the decay of the mean horizontal kinetic energy $E$. The energy and the time are normalized with $U^{2}$ and $M / U$ respectively. From now on we will use the notation $t^{*}$ for the non-dimensional horizontal advective time: $t^{*}=t U / M$. Using this normalization, the data collapse fairly well, indicating that the turbulent kinetic energy depends only on the velocity and the mesh of the rake. This similarity suggests universality of decay, for strong enough stratification, with very little dependence on the stratification. This result was also observed in the numerical experiments of Riley et al. (1981) for Froude numbers smaller than 1.

Figure 4 shows an asymptotic power law of decay

$$
E \equiv\left\langle u_{x}^{2}+u_{y}^{2}\right\rangle / 2=\left\langle u^{2}\right\rangle \simeq 0.36 U^{2} t^{*-1.3} .
$$

The exponent -1.3 is similar to that found for the horizontal kinetic energy in the very early decay of stratified turbulence by Lienhard \& Van Atta (1990), Yoon \& Warhaft (1990) and in numerical simulation by Staquet \& Godeferd (1998). It was also obtained for late times, after the collapse, by Fincham et al. (1996). Surprisingly, this decay exponent, -1.3 , is also similar to that observed for decaying three-dimensional isotropic homogeneous turbulence. For instance Comte-Bellot \& Corrsin (1966) found a decay exponent of the kinetic energy equal to -1.26 , while Warhaft \& Lumley (1978) found -1.34 . This contrasts with the dependence of energy dissipation on Reynolds 
(a)

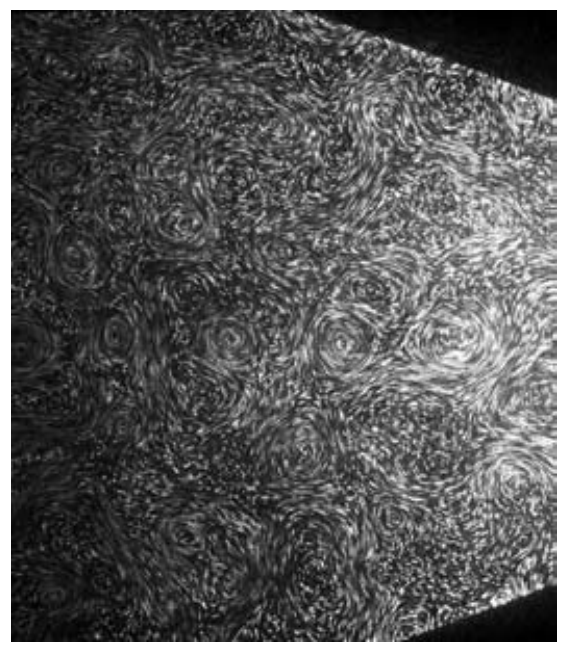

(c)

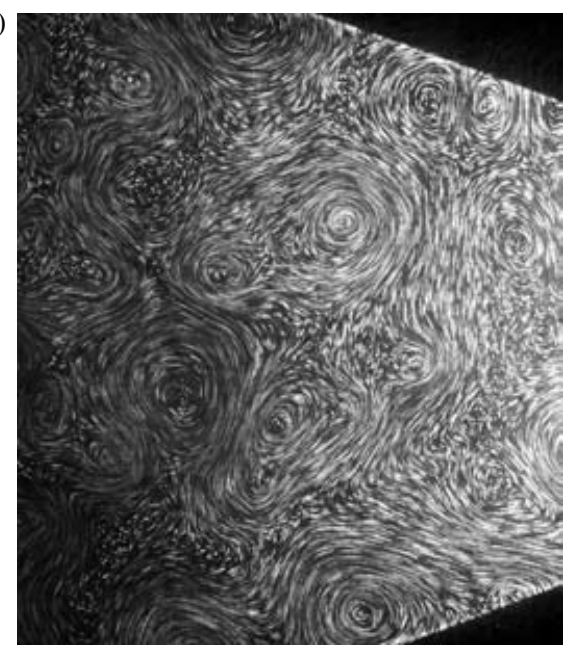

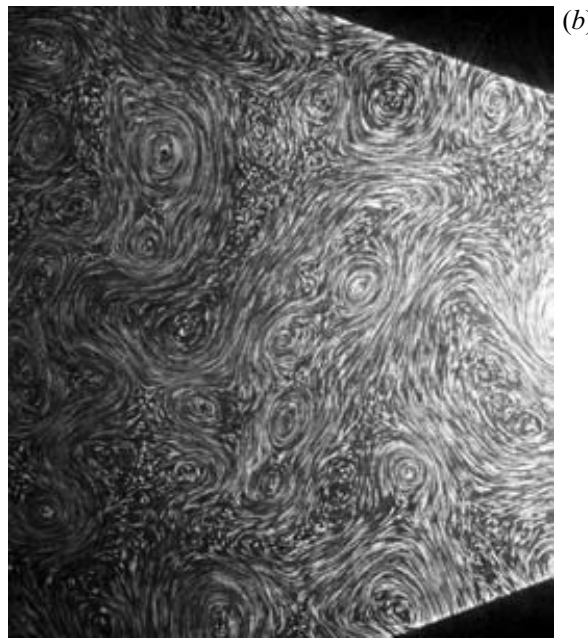

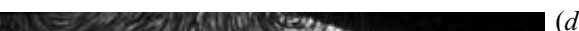

$(d)$

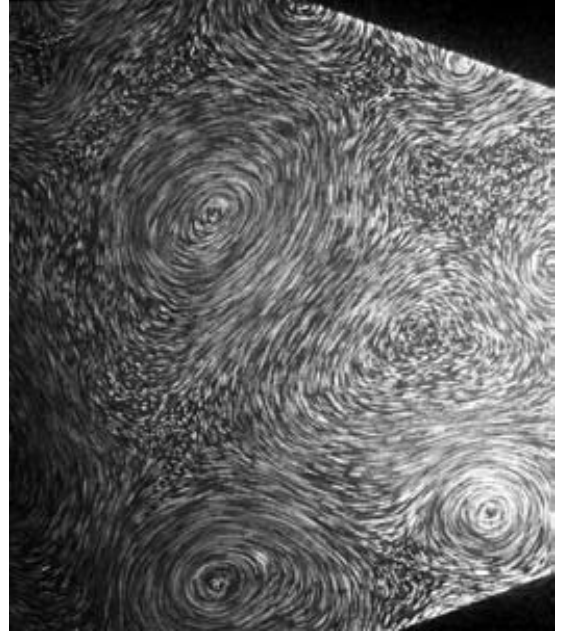

FIGURE 3. Streak photographs of an evolving, grid-generated turbulent flow. Photographs were taken 74, 137, 205 and $407 \mathrm{~s}$ after $t_{0}$. The grid was towed from right to left. $R e_{M}=18000$, $\mathrm{Fr}_{M}=0.17$.

number in purely two-dimensional turbulence leading to its absence in the limit of infinite $R e$.

Because of the internal gravity waves and the instantaneous curvature of the isopycnals, the motion is not purely horizontal but we can show that the contribution of the vertical velocity to the total kinetic energy is very weak. The vertical velocity can be evaluated through the continuity equation, that gives in Fourier space, for $k_{z} \neq 0$

$$
\hat{u}_{z}(\boldsymbol{k})=\frac{\boldsymbol{k}_{h} \cdot \hat{\boldsymbol{u}}_{h}(\boldsymbol{k})}{k_{z}} .
$$

For $k_{z}=0$ we arbitrarily choose $\hat{u}_{2}\left(k_{x}, k_{y}, 0\right)=0$. This choice consists of removing all the waves of frequency $N$ or to the assumption that there is no mean vertical motion in any vertical line. This evaluation gives a good approximation of the contribution of the vertical velocity to the total energy. Figure 5 compares this vertical energy $E_{v}$ 


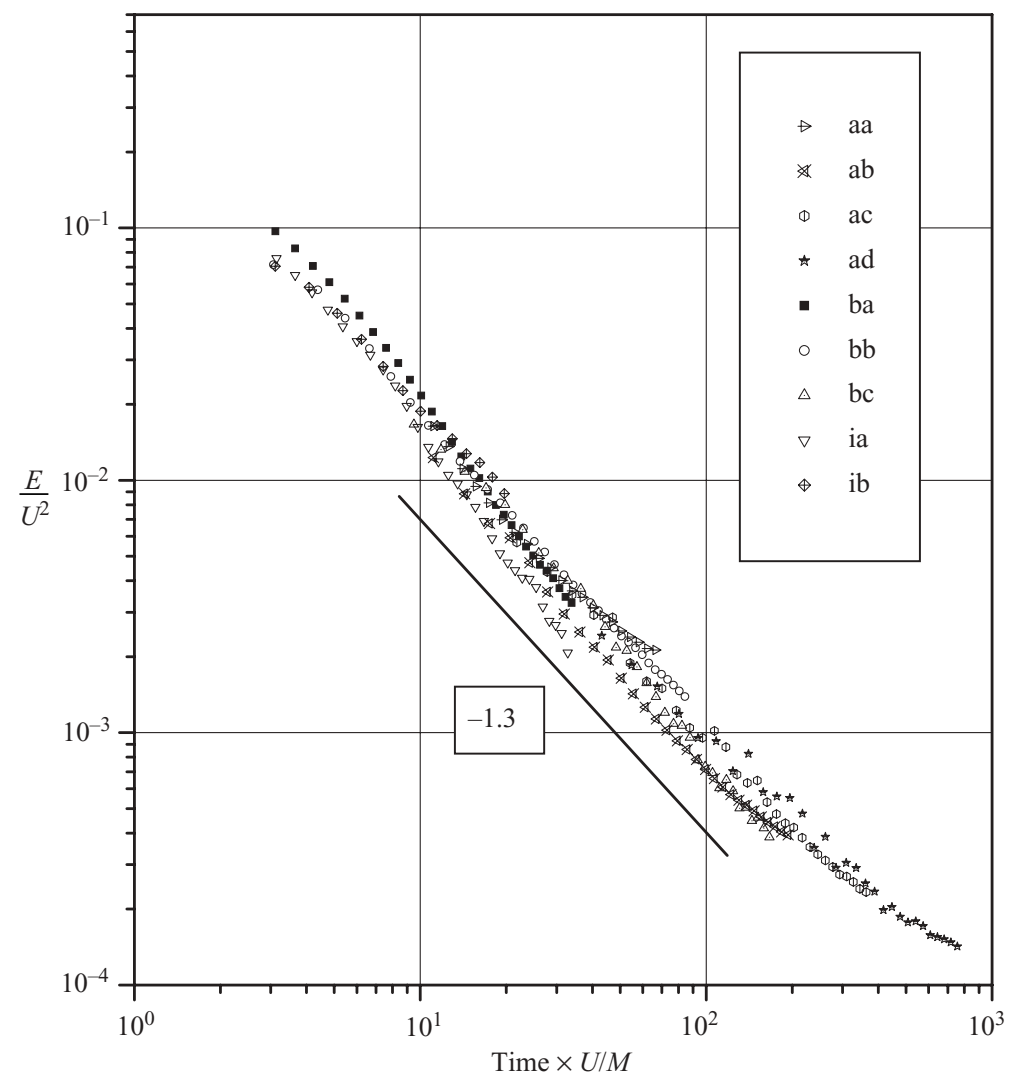

FIGURE 4. Decay of the normalized mean kinetic energy $E$, averaged over the volume of the experiment. Experiments are listed in table 1.

to the horizontal energy, $E_{h}$. Clearly, the contribution of $E_{v}$ is negligible for all the experiments (less than $1 \%$ ) and decreases with time (less than $0.02 \%$ at the end of the experiment). The horizontal kinetic energy can thus be considered to be the total kinetic energy of the flow.

\subsection{Laminar initial wake}

For low $U$, immediately after the passage of the rake, a regular von Kármán type of vortex shedding is observed in the horizontal plane (see figure 6).

Behind each plate of the rake, we observe the formation of columnar vortex pairs, initially straight and uniform along the vertical. These columnar vortices rapidly exhibit symmetric sinusoidal deformation as can be seen in figure 7 . The shed vortices decorrelate vertically, eventually producing multiple layers of eddies. The vertically uniform flow is thus unstable and no straight columnar vortices persist.

The mechanisms of layer formation and the selection of their vertical scale is still not clearly understood. Spontaneous generation of layers by a so-called zig-zag instability is proposed by Billant \& Chomaz (2000b). A vertical columnar vortex pair created by a long pair of vertical flaps is sliced into layers of 'pancake' dipole by this instability. The vertical scale selected by this mechanism is the most amplified wavelength of the instability and is proportional to $U / N$, the buoyancy length scale. Flow decorrelation and layering of eddies can also be induced by a weak, vertically dependent, horizontal velocity as discussed by Majda \& Grote (1997). The vertical 


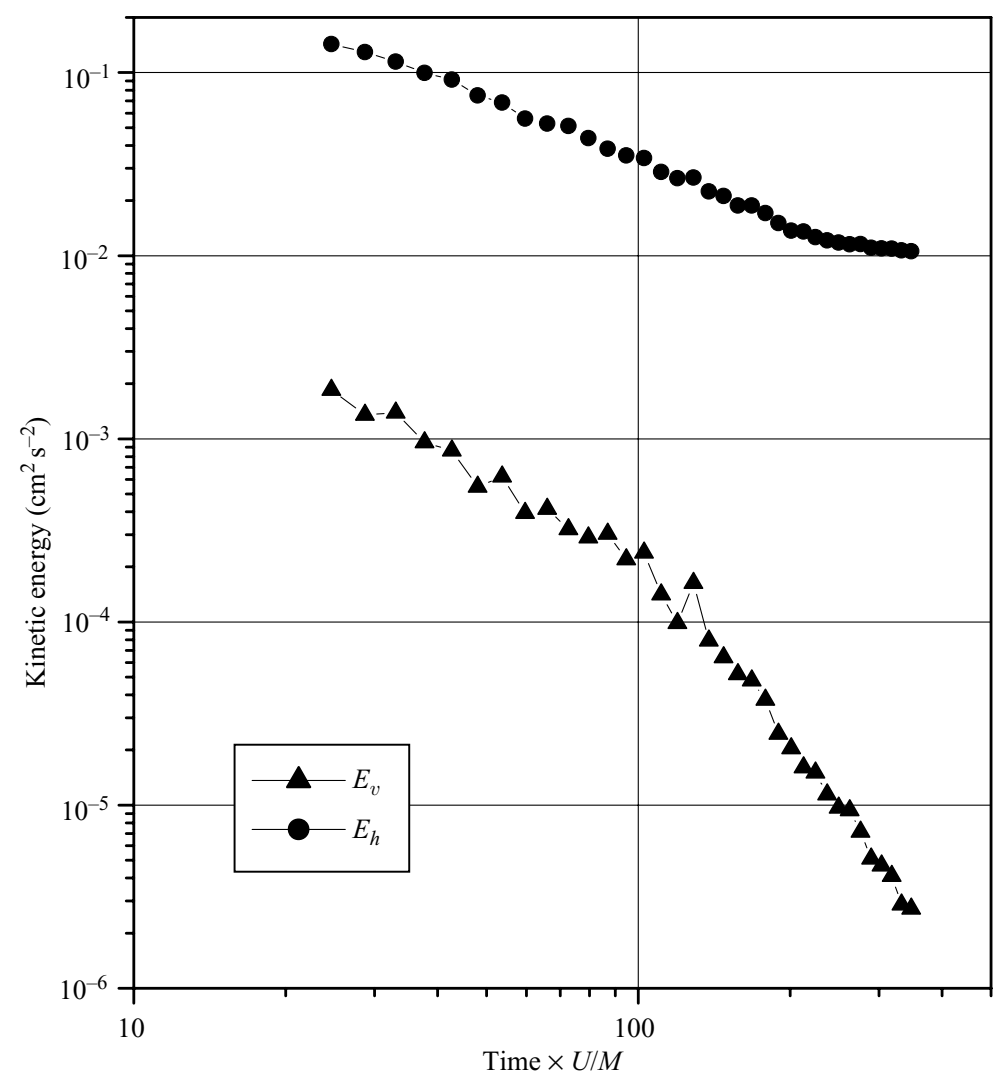

FIGURE 5. Comparison of the horizontal kinetic energy $E_{h}$ and the estimated vertical kinetic energy $E_{v} . R e_{M}=18000, F r_{M}=0.17$.

scale is then set by the weak external shear. We observed that imperfections in the density profile (stronger or weaker stratification) influence the layer positions. In any case, we shall see in $\S 3.5$ that the vertical scale in the turbulent regime is not influenced by this initial behaviour.

\subsection{Turbulent initial wake}

For higher towing speeds, i.e higher Reynolds numbers, $R e_{M}=O\left(10^{4}\right)$, and higher Froude numbers, the flow immediately behind the rake is highly three-dimensional, as shown by direct observation. This active turbulence rapidly collapses, after $N t \sim 30$, under the effect of stratification as the vertical velocity component is suppressed. It results in multiple layers of quasi-horizontal vortical structures.

The structures that emerge after the collapse of three-dimensional turbulence are such that the gradient Richardson number, $R i=N^{2} /\left(\partial u_{x} / \partial z\right)^{2}$, is found to be greater than the critical value for stability: $R i_{c}=1 / 4$. So no overturning instability is expected and the fluid remains stable with respect to the vertical shear as the Richardson number continues to increase with time. Figure 8 shows the probability distribution function of the vertical turbulent Froude number $F r_{v}=\left(\partial u_{x} / \partial z\right) / N$ for different times. The vertical Froude number is directly related to the Richardson number by $\mathrm{Fr}_{v}^{-2}=\mathrm{Ri}$ so the critical Richardson number correspond to a critical value of $F r_{v}$ equal to 2 . It appears that by the time of our first measurement $F r_{v}$ does not exceed the critical value, so the vertical shear is not strong enough to produce overturning. At early 


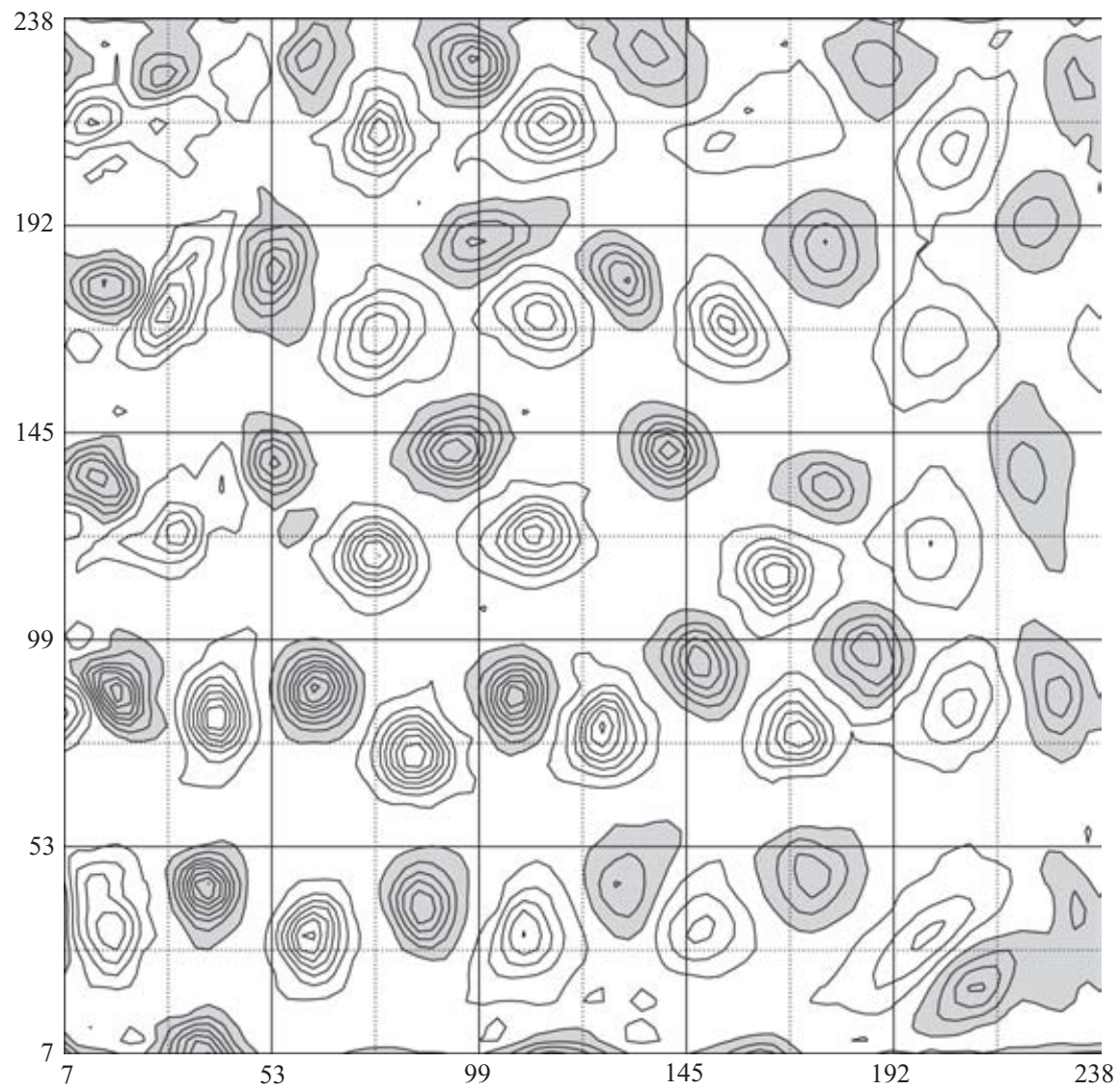

FIGURE 6. Initial wake. Isolines of the vertical component of vorticity observed in an horizontal plane. $\omega_{z}(x, y) . \Delta \omega_{z}=0.02 \mathrm{~s}^{-1}$. The axes are in $\mathrm{cm}$. The negative vertical vorticity is shaded grey. The grid was towed from right to left. $R e_{M}=2550, F r_{M}=0.02, t=280 \mathrm{~s}$.

times, before the start of the measurements, Kelvin-Helmholtz-type roll-up is evident, as seen in figure 6 of Fincham et al. (1996), which corresponds to $N t \sim 10$.

The Gaussian-like shape of the distribution of the vertical gradient indicates that there is no tendency for the flows to develop strong vertical gradients in small spatial regions. We do not observe any intermittency of the horizontal vorticity field. Similar observations of a Gaussian-like distribution of vertical shear in strongly stratified turbulence (after the collapse) were also made in numerical simulations of freely evolving stratified turbulence by Metais \& Herring (1989) and forced stratified turbulence by Herring \& Metais (1989).

\subsection{Fully established stratified turbulence}

In all cases, laminar or turbulent initial wake, the final stage of the flow is characterized by quasi-horizontal motion, organized into horizontal layers (see figures 9 and 10).

As seen in figure 9 the flow contains patches of vertical vorticity localized in space and bounded by horizontal vortex sheets. These horizontal vortex sheets are associated with the strong vertical shear of the quasi-horizontal motion (see figure 10). The horizontal r.m.s. vorticity $\left\langle\omega_{h}^{2}\right\rangle^{1 / 2}$ contained in these thin sheets is much stronger than the vertical r.m.s. vorticity $\left\langle\omega_{z}^{2}\right\rangle^{1 / 2}$ of the larger horizontal structures themselves, 


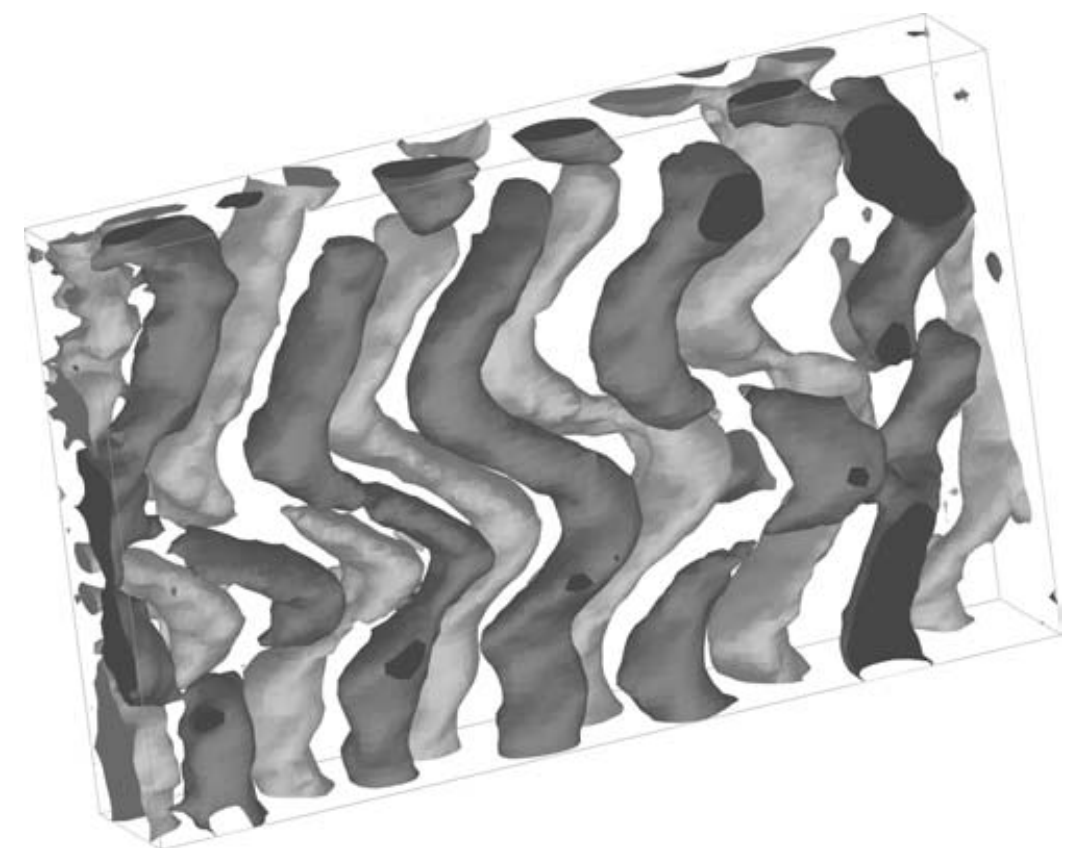

FIGURE 7. Initial three-dimensional instability of the laminar wake represented in terms of isosurfaces of the vertical component of vorticity $\omega_{z}(x, y, z)$. The positive vertical vorticity is in dark grey and negative in light grey. The vertical dimension is magnified four times with respect to the horizontal. The grid was towed from right to left. $R e_{M}=2550, F r_{M}=0.02$, $t=280 \mathrm{~s}$.

as can be seen in figure 11. This plot of the contribution of the three components of the vorticity $\left(\omega_{x}, \omega_{y}, \omega_{z}\right)$ to the total enstrophy $\Omega=\frac{1}{2}\left\langle\omega^{2}\right\rangle$ shows clearly that the horizontal vorticity contributes more than $95 \%$ to the total enstrophy, and this contribution increases with Reynolds number, consistent with the findings of Fincham et al. (1996). The strongly sheared regions are highly dissipative and the energy contained in the pancake eddies is expected to be dissipated primarily in the thin vortex sheets present at their edges.

\subsection{Length scale evolution}

To elucidate the results on energy decay and the structure of the flow, we describe here the evolution of the length scales. We first measure the integral length scales. The horizontal integral scale $L_{h}$ is obtained from the longitudinal auto-correlation function of the horizontal velocity by integrating up to its first zero. For the vertical integral scale, we perform the same analysis on the vertical auto-correlation function of the horizontal velocity (it is therefore a transverse auto-correlation function). The horizontal velocity is indeed the dominant component.

Alternatively, the Taylor microscale provides "a measure of the average dimension of the eddies that are mainly responsible for dissipation" (Hinze 1975). It is obtained from the auto-correlation function of the velocity, as the point where the osculation parabola at the origin meets the horizontal axis. Like for the integral scales, we shall determine the horizontal Taylor microscale, $\lambda_{h}$, using the longitudinal auto-correlation function, and the vertical one, $\lambda_{v}$, using the transverse auto-correlation function of the horizontal velocity. Note that for isotropic turbulence, the longitudinal and transverse 


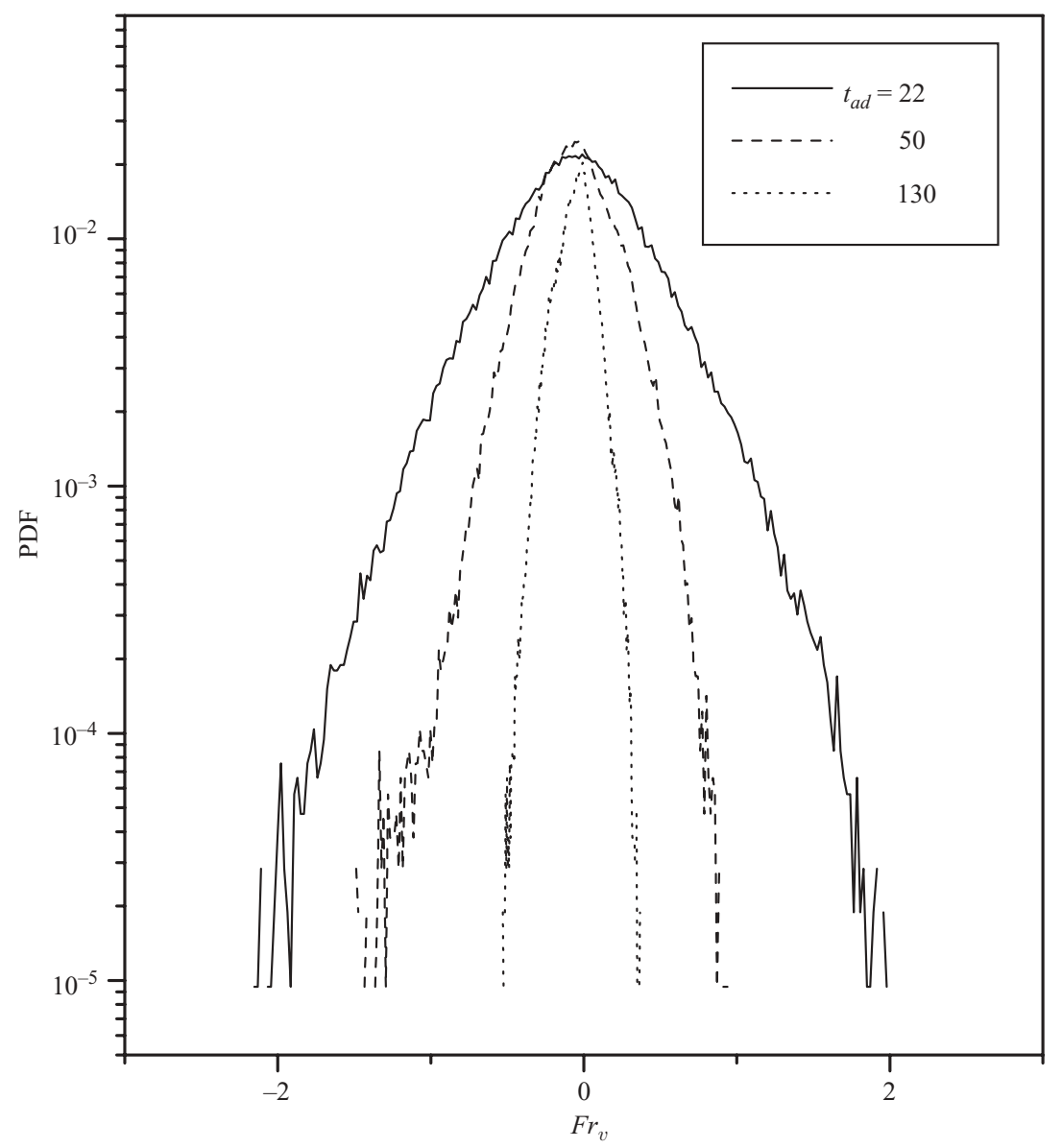

FIGURE 8. Probability distribution of the vertical turbulent Froude number, $F r_{v}$, at different stages of the experiment. $R e_{M}=18000, F r_{M}=0.34$.

auto-correlations function coincide at the origin so that they yield the same Taylor microscale.

\subsubsection{Horizontal scales}

Figure 12 shows the evolution of $L_{h}$ with time for various experiments. The BruntVäisälä frequency for experiments $\mathrm{ba}, \mathrm{bb}, \mathrm{bc}, \mathrm{bd}$ and be is $N=0.52 \mathrm{~s}^{-1}$ while $N=$ $0.26 \mathrm{~s}^{-1}$ for experiments $\mathrm{ia}$, ib and id. Figure 12 shows that the typical size of the energy-containing eddies grows with time as previously observed (see figure 3 ). The collapse of the curve is not very good but no obvious dependence of $L_{h}$ on stratification can be seen. A fit

$$
L_{h} / M \simeq 0.25 t^{* 0.5}
$$

can be proposed. For late times, the increase of $L_{h}$ slows down, and this is probably due to confinement by the channel width.

Figure 13 shows the evolution of the horizontal Taylor microscale $\lambda_{h}$, for various experiments. It grows with time, approximately as a power law $t^{0.37}$. The collapse of the curves indicates that $\lambda_{h}$ does not depend on the initial condition or on the 


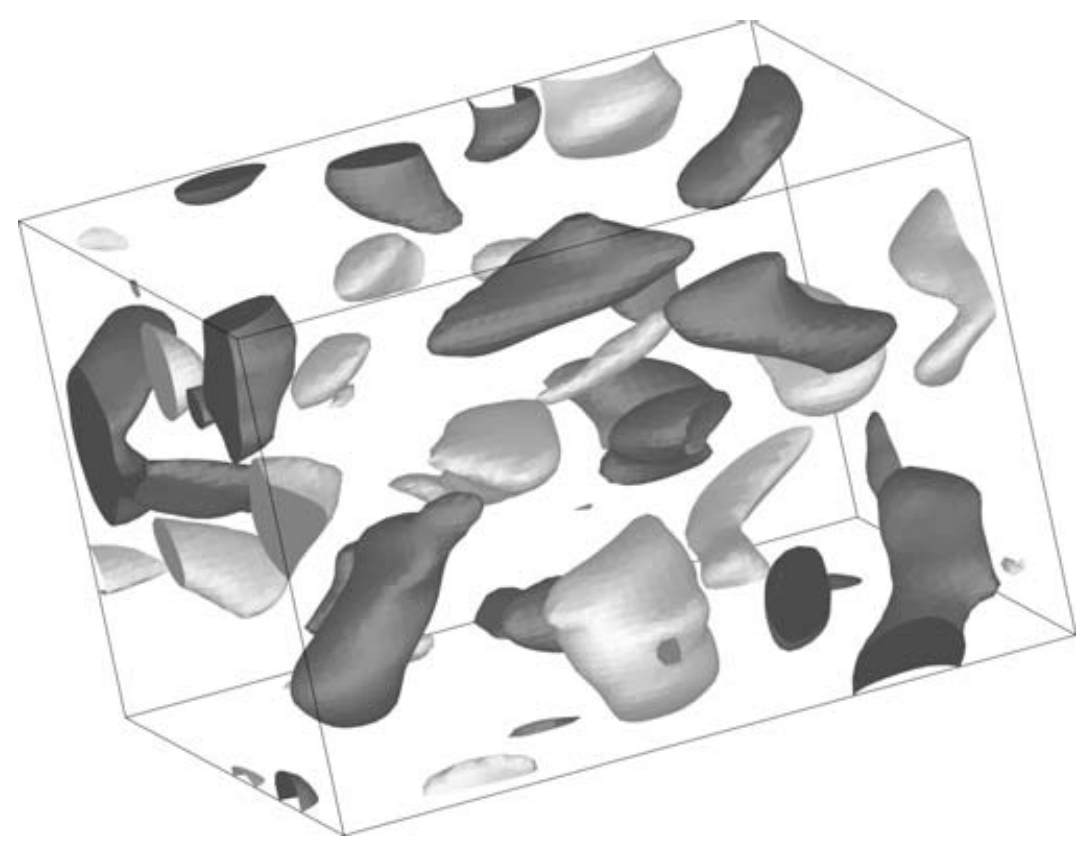

FIGURE 9. Three-dimensional structure of the vertical vorticity. The dark regions represent blobs of positive $\omega_{z}$ while the white regions represent blobs of negative $\omega_{z}$. The vertical dimension is magnified three times. $\operatorname{Re}_{M}=9000, F r_{M}=0.09, N=0.52, t=700 \mathrm{~s}$.

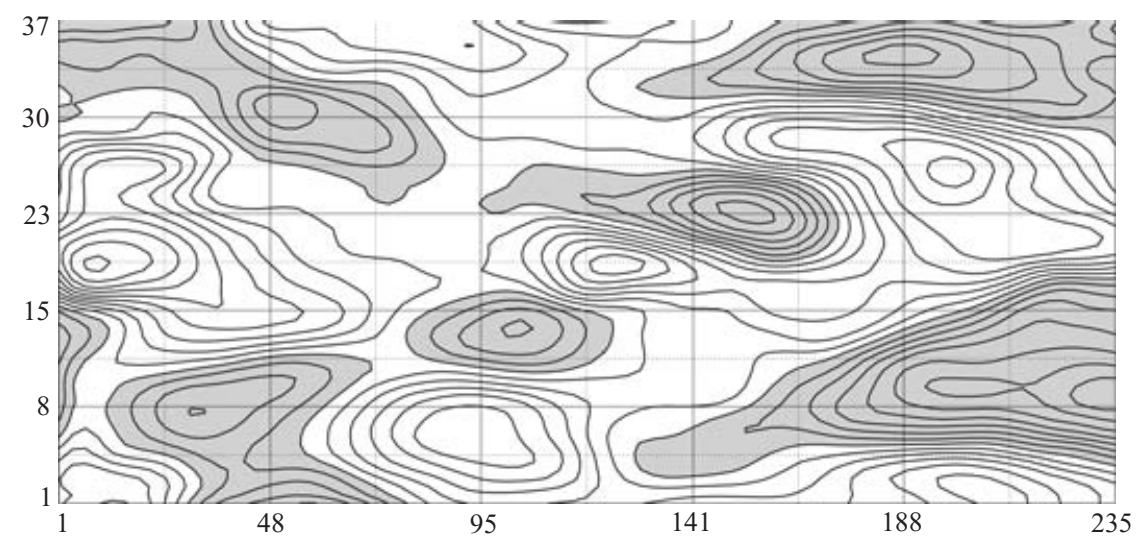

FIGURE 10. Isolines of the transverse velocity $u(y, z)$ in a vertical slice. The negative part of $u(y, z)$ is represented in grey and the positive part in white. The vertical dimension is magnified three times and the axes are in $\mathrm{cm} . R e_{M}=9000, F r_{M}=0.09, N=0.52 t=700 \mathrm{~s}$.

stratification. For very late times, the plot becomes scattered however, and this is probably due to the poor statistics obtained when few eddies remain in the view field.

The r.m.s. of the vertical vorticity can be related to the horizontal Taylor microscale $\lambda_{h}$ and the kinetic energy $\left\langle u^{2}\right\rangle$ through the relation:

$$
\lambda_{h}^{2}=\frac{\left\langle u^{2}\right\rangle}{\left\langle\omega_{z}^{2}\right\rangle} .
$$




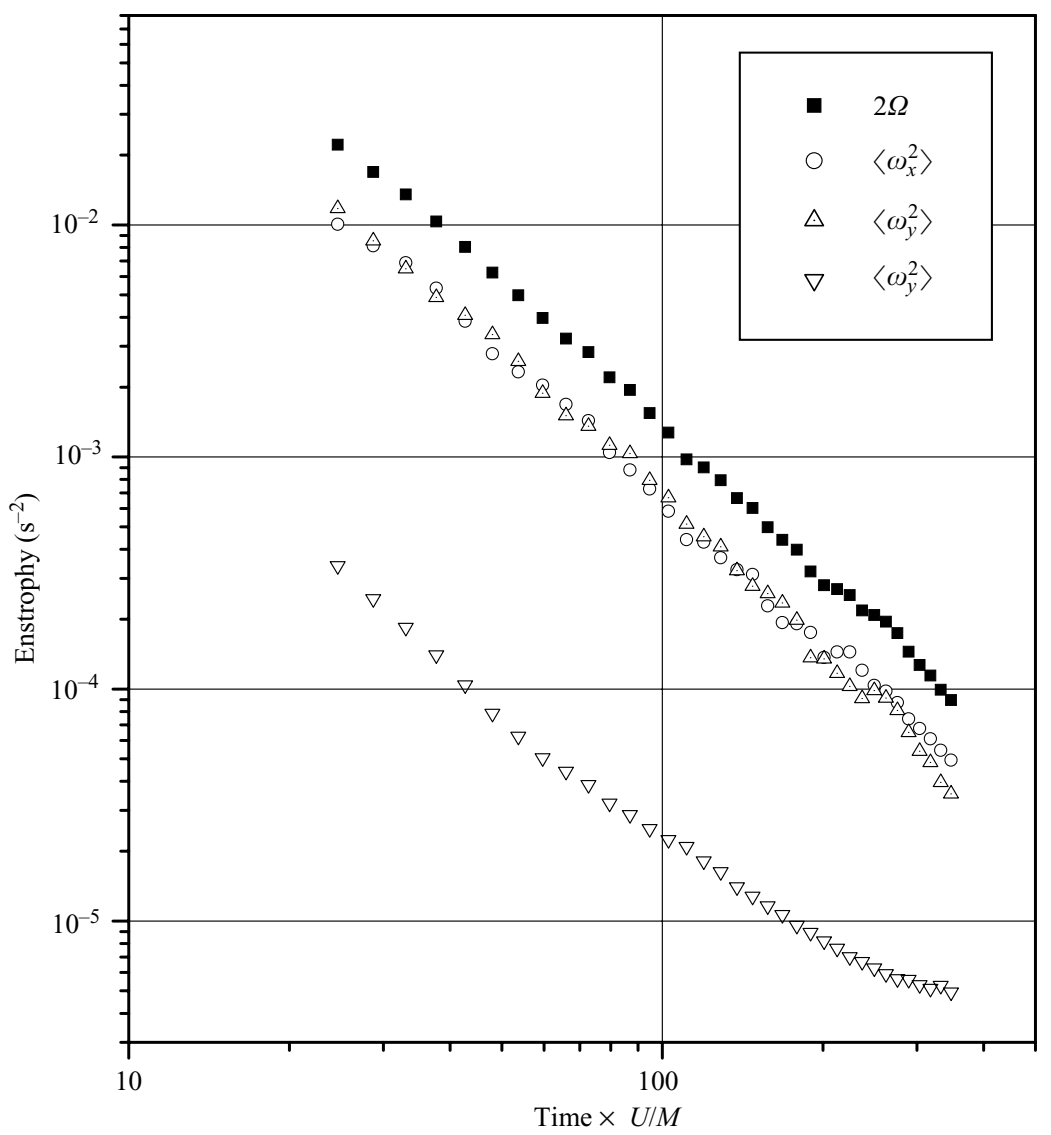

FIGURE 11. Contribution of the three components $\left(\omega_{x}, \omega_{y}, \omega_{z}\right)$ of the vorticity to the total enstrophy $\Omega$. $R e_{M}=18000, F_{M}=0.17, N=0.52$.

We have shown that $\left\langle u^{2}\right\rangle \sim t^{-1.3}$ and $\lambda_{h} \sim t^{0.37}$. Moreover both are independent of the initial conditions and in particular of stratification. Therefore $\left\langle\omega_{z}^{2}\right\rangle$ should also be independent of stratification and behave like $t^{-2}$. The good agreement with this prediction is shown in figure 14 where the evolution of the normalized vertical vorticity is plotted against the advective time. The collapse is fairly good and confirms the predicted behaviour.

\subsubsection{Vertical scales}

Figure 15 shows the evolution of the vertical integral scale. $L_{v}$ grows monotonically with time as the vertical extent of the structures increases by viscosity. In some cases, for early times a decrease of $L_{v}$ is observed. This phenomenon is associated with the loss of coherence of the columnar vortices observed in the laminar initial wake just behind the rake, as mentioned in $\S 3.4$. An interesting fact is that in these cases, $L_{v}$ first decreases and then starts to increase following exactly the same law (independent of the initial conditions) as the other experiments. This observation suggests a process of adjustment of the vertical size of the pancakes by the turbulent dynamics: the horizontal advection tends to break the columnar vortices while viscous diffusion continually increases the vertical scale. 


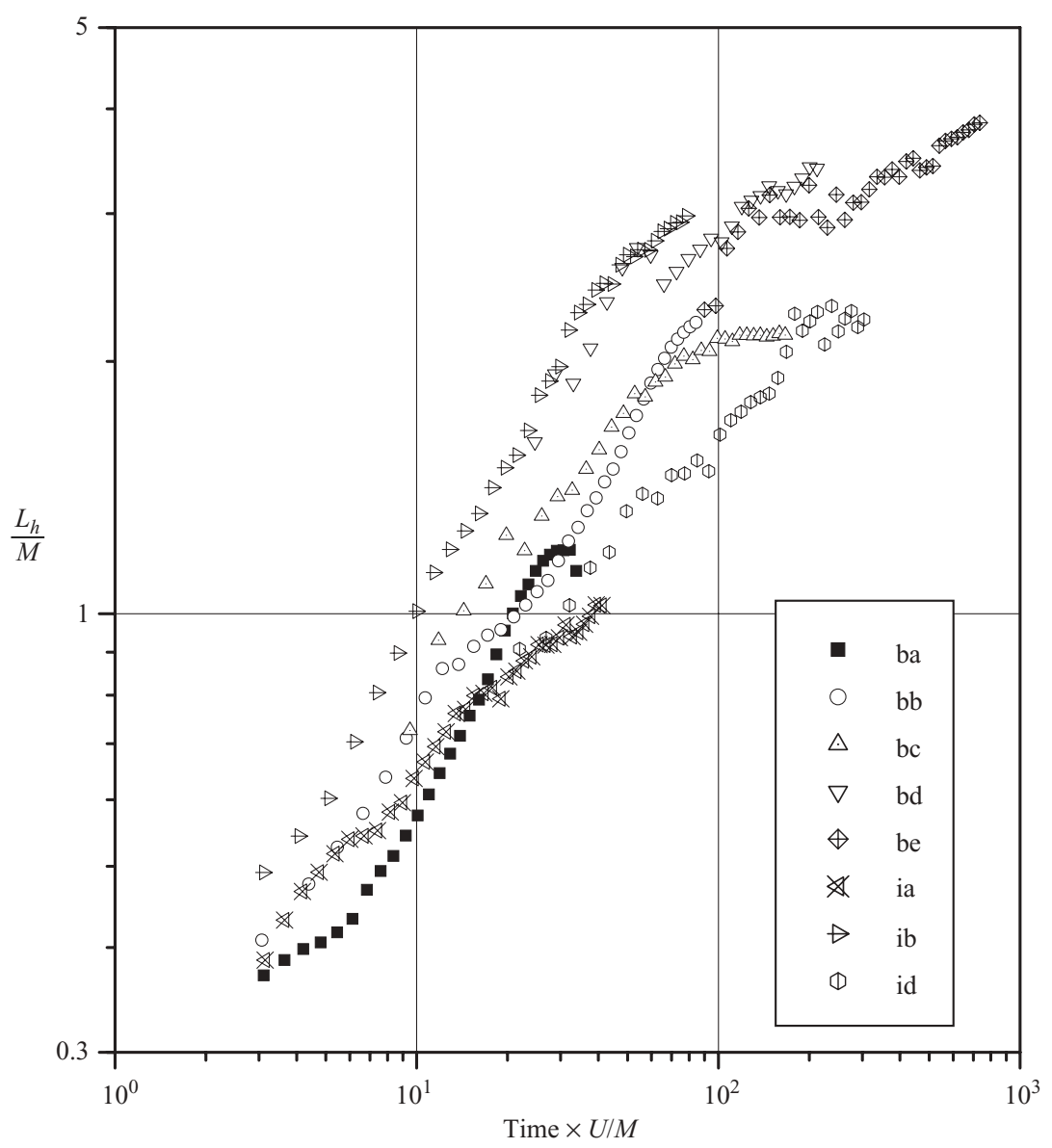

FIGURE 12. Evolution of the horizontal integral length scale, $L_{h}$. Experiments are listed in table 1 .

The law $L_{v} \simeq 2 \sqrt{v t}$ suggests that the vertical scale is controlled by viscosity and evolves following a diffusive process. In particular, no dependence on $U / N$ is found in the turbulent regime. Billant \& Chomaz (2000b) argue that the layering scale in the fully turbulent regime should be the same as the most amplified wavelength of the zig-zag instability and thus be proportional to the buoyancy scale $U / N$. The results of the present set of experiments do not confirm this. In their experiments the dipolar vortices generated by the breakdown of the columnar dipole are free to evolve without horizontal interactions in the quiescent ambient fluid. In our experiments each vortex column immediately feels the presence of the neighbouring columns and any preferred wavelengths will have to compete with the vertical shearing induced by the flow field.

The aspect ratio of the structures, based on the ratio of the integral scales, $\alpha=L_{v} / L_{h}$ ranges between 0.05 and 0.4 . These low values of $\alpha$ are consistent with previous observations of pancake eddies. For example, Bonnier et al. (2000) measure $\alpha \sim 0.4$, Fincham et al. (1996) $\alpha \sim 0.3$, and for the vortex dipole, Voropayev, Afanasyez \& van Heijst (1995) find $\alpha \sim 0.4$ whereas Flor, van Heijst \& Delfos (1995) find $\alpha \sim 0.3$. 


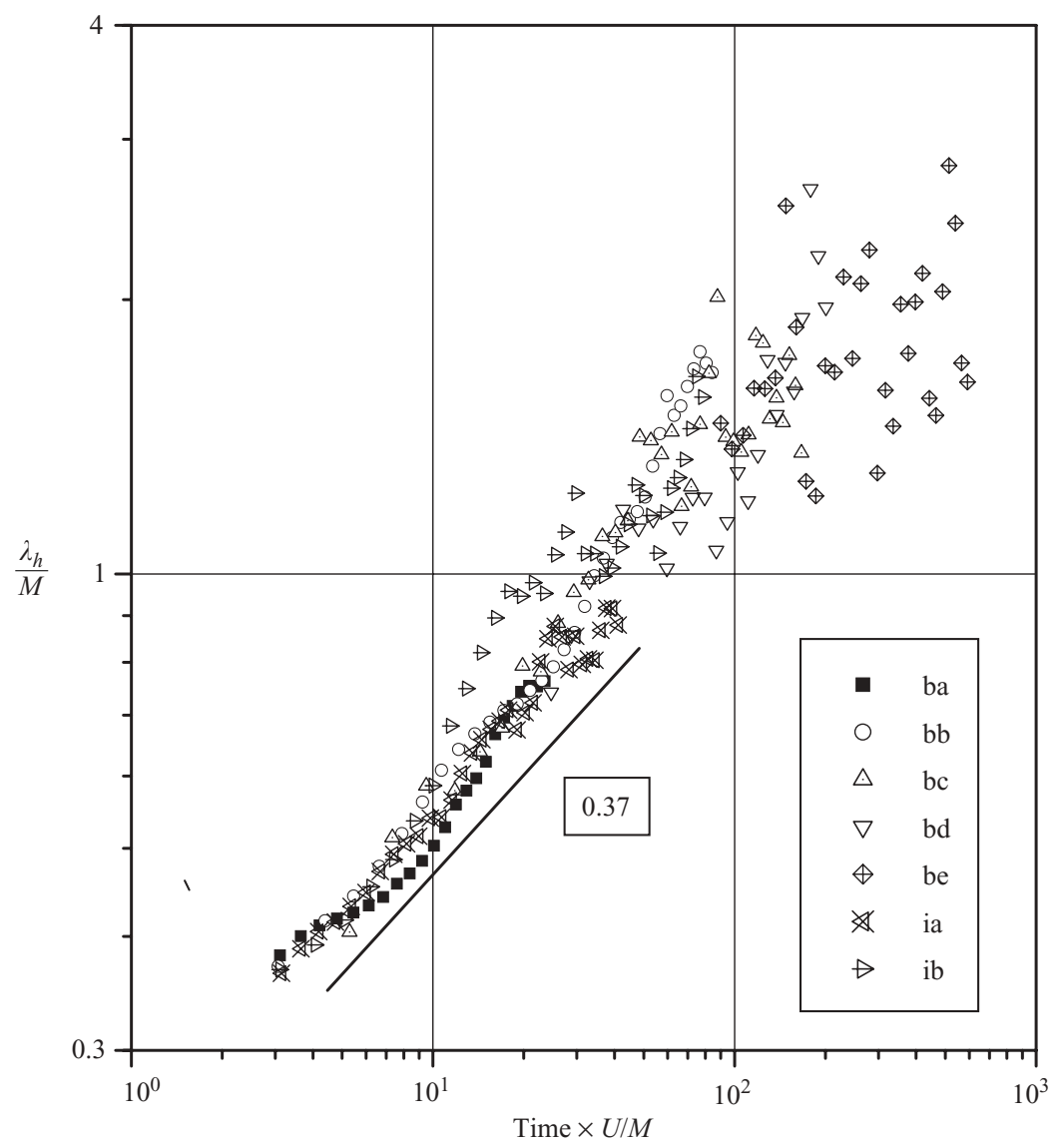

FIgURE 13. Evolution of the horizontal Taylor microscale $\lambda_{h}$. Experiments are listed in table 1.

The vertical Taylor microscale is plotted in figure 16 in both dimensionless and physical units. We note that this scale approximately follows a law

$$
\lambda_{v} \simeq 2 \sqrt{v t},
$$

so that $L_{v} \simeq \lambda_{v}$ : most of the energy is at the Taylor microscale. Like the corresponding integral scale, the vertical Taylor microscale is not significantly influenced by the initial condition (mesh size), the velocity of the rake or the stratification. It is entirely set by viscosity. Accordingly the non-dimensional result,

$$
\lambda_{v}^{*} \simeq 2 . t^{* 1 / 2} \operatorname{Re}_{M}^{-1 / 2}
$$

does not collapse onto a single curve (figure 16a): it depends on the Reynolds number.

This result for the vertical Taylor microscale can be explained by relating it to the energy dissipation. Indeed this scale is theoretically related to vertical gradient of the horizontal velocity $\left\langle\left(\partial u_{x} / \partial z\right)^{2}\right\rangle$ by

$$
\lambda_{v}^{2}=\frac{\left\langle u^{2}\right\rangle}{\left\langle\left(\partial u_{x} / \partial z\right)^{2}\right\rangle} .
$$

The agreement between this determination of $\lambda_{v}$ and its determination from the vertical correlation function has been checked and the deviation is found to be less 


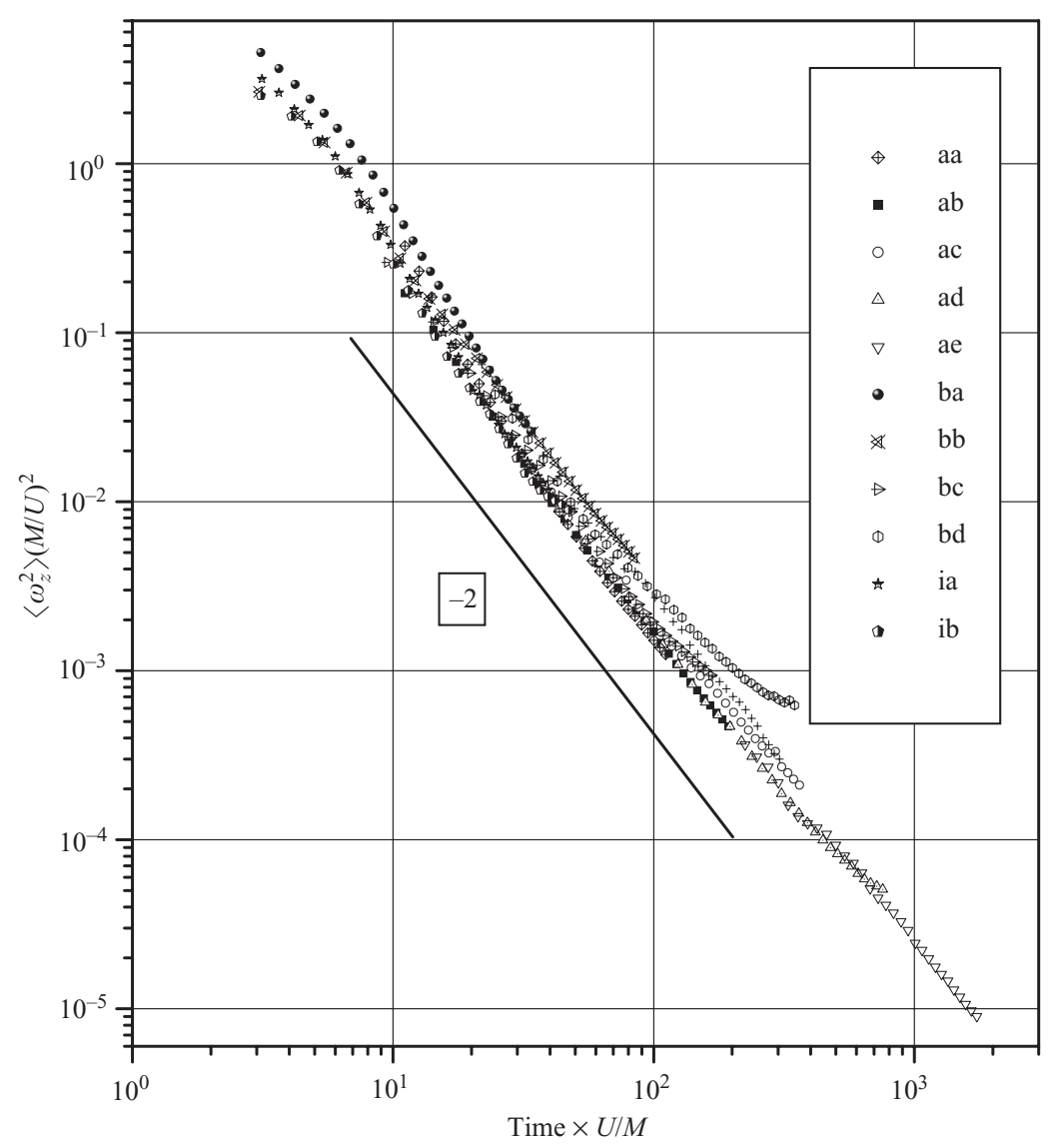

FiguRE 14. Decay of $\left\langle\omega_{z}^{2}\right\rangle$. Experiments are listed in table 1.

than $5 \%$. Assuming that the energy dissipation is entirely due to the vertical velocity gradients, the dissipation rate of kinetic energy $\epsilon$ can be approximated, following Fincham et al. (1996), as

$$
\epsilon \simeq 2 \nu\left\langle\left(\frac{\partial u_{x}}{\partial z}\right)^{2}\right\rangle
$$

(horizontal isotropy is assumed). A more precise justification will be given in $\S 6$. Using (3.7) and (3.8), we obtain

$$
\lambda_{v}^{2}=2 v \frac{\left\langle u^{2}\right\rangle}{\epsilon}
$$

if energy is lost only to viscous dissipation. Since $\left\langle u^{2}\right\rangle \sim t^{-1.3}$ and $\epsilon=-\mathrm{d}\left\langle u^{2}\right\rangle / \mathrm{d} t=$ $-1.3\left\langle u^{2}\right\rangle t^{-1}$, we have $\lambda_{v} \simeq 1.25 \sqrt{v t}$, close to the measured result (3.5).

We can evaluate the anisotropy of the flow based on the ratio of the vertical to the horizontal Taylor microscales. We have shown that $\lambda_{h}^{*} \sim t^{* 0.37}$ and is independent of the Reynolds and Froude numbers, while $\lambda_{v}^{*} \sim R e_{M}^{-1 / 2} t^{* 1 / 2}$. Therefore

$$
\frac{\lambda_{v}^{2}}{\lambda_{h}^{2}} \sim R e_{M}^{-1} t^{* 0.26} \quad \text { or } \quad \frac{\left\langle\omega_{z}^{2}\right\rangle}{\left\langle\omega_{h}{ }^{2}\right\rangle} \sim R e_{M}^{-1} t^{* 0.26} .
$$




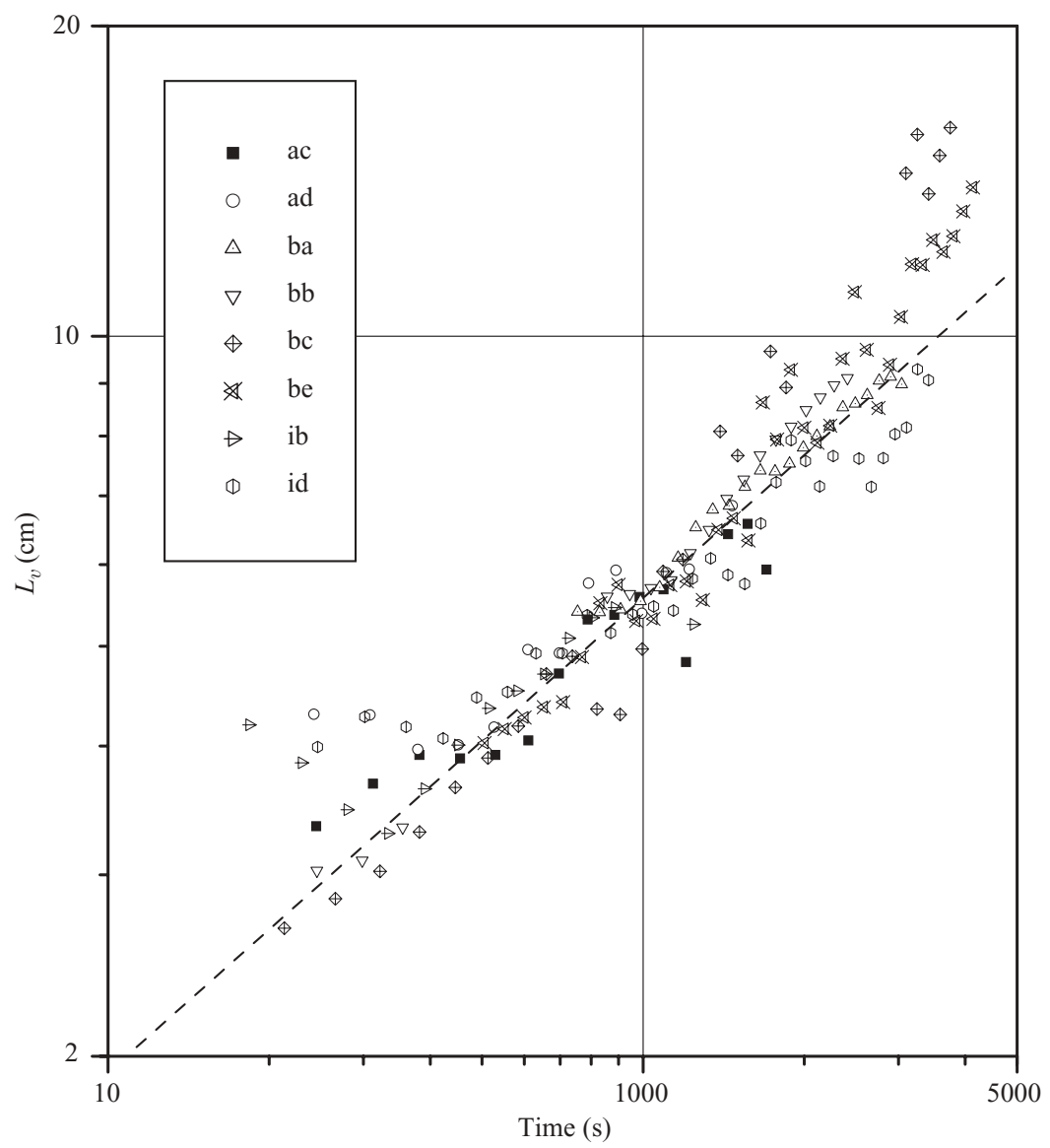

Figure 15. Evolution of vertical integral scale $L_{v}$. The dashed line represents the function $2 \sqrt{v t}$. Experiments are listed in table 1 .
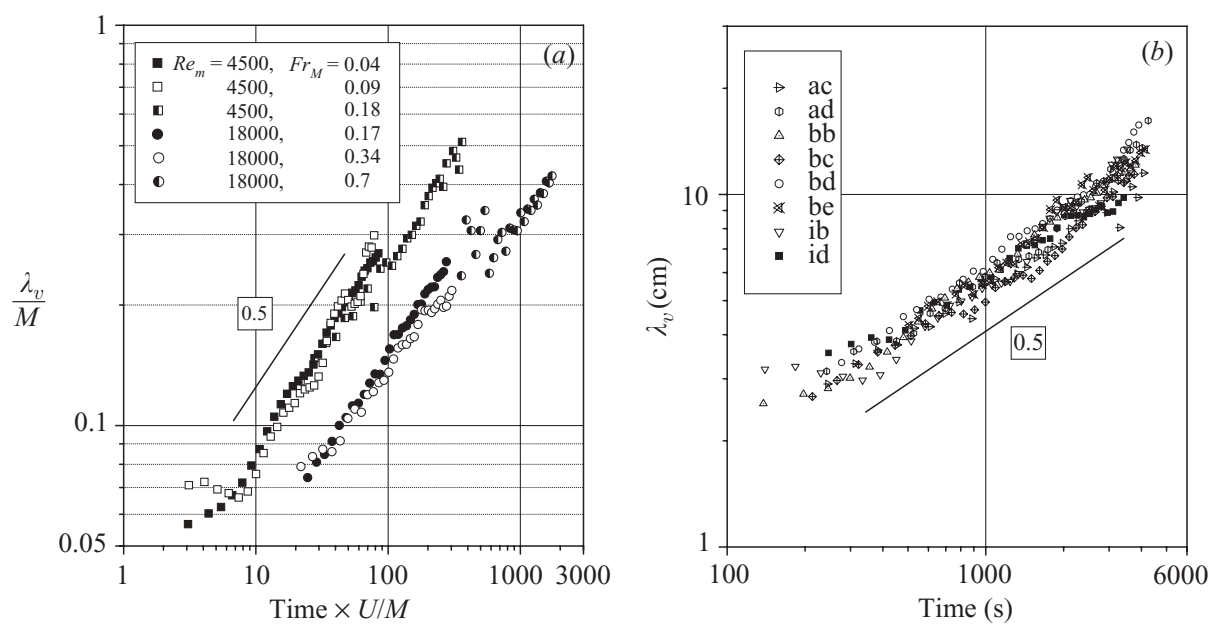

FiguRE 16. Evolution of vertical Taylor microscale for various experiment. $(a)$ normalized $\lambda_{v}$ versus $t^{*}$. (b) $\lambda_{v}$ versus time. Experiments are listed in table 1. 


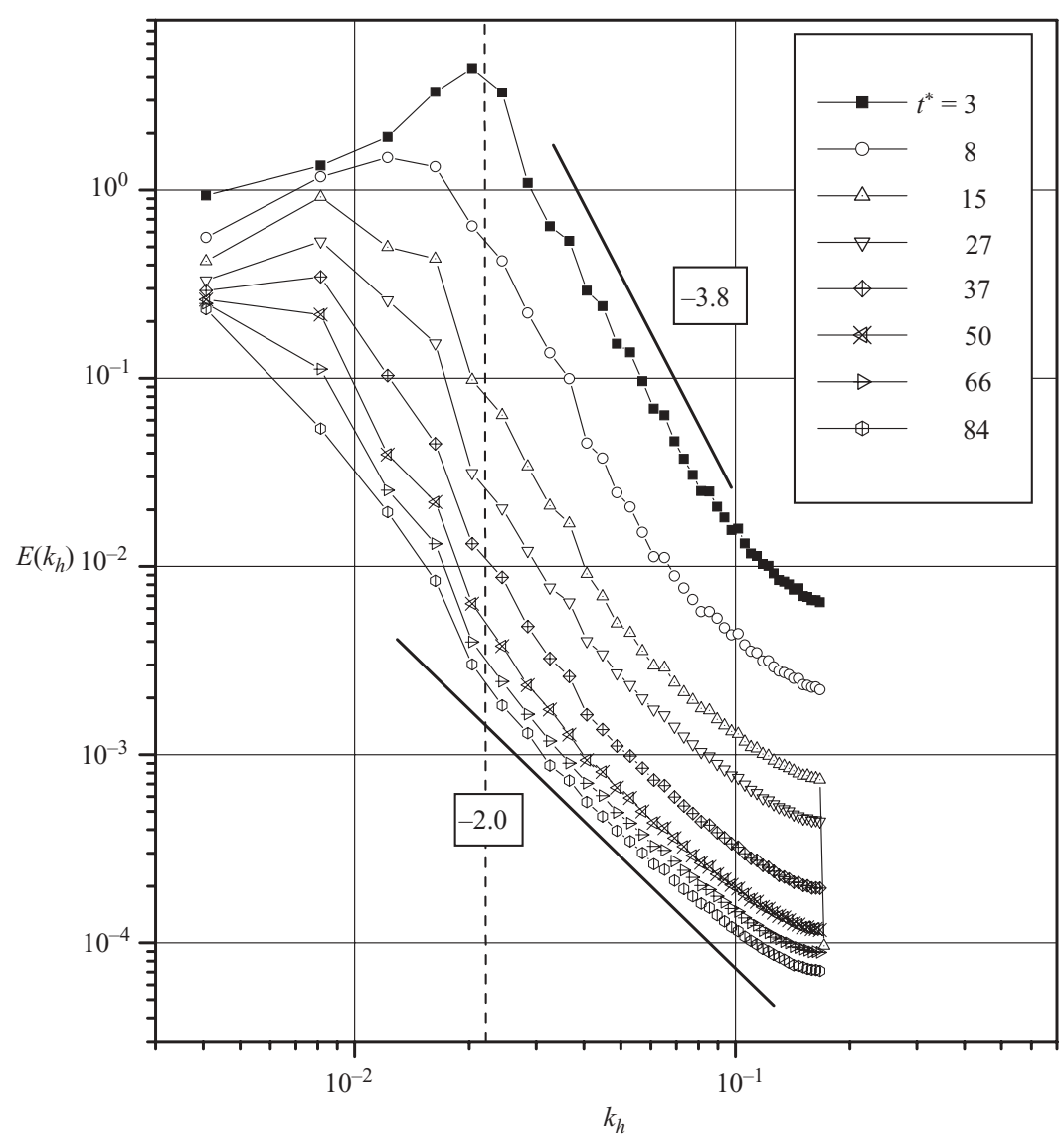

FIGURE 17. Two-dimensional energy spectra at different stages of the experiments. The dashed line respresents the wave number corresponding to the mesh. $\operatorname{Re}_{M}=4500, F_{M}=0.09$, $M=45 \mathrm{~cm}$.

The aspect ratio based on the Taylor microscale does not depend on the stratification and grows slowly with time (as $\left.t^{0.13}\right)$.

\section{Spectral analysis}

\subsection{Horizontal spectra}

The two-dimensional horizontal energy spectrum is calculated as a function of the horizontal wavenumber $k_{h}=\left(k_{x}^{2}+k_{y}^{2}\right)^{1 / 2}$. A two-dimensional fast Fourier transform of the horizontal velocity field is performed in each horizontal plane of the measurement volume. Then a circumferential average at radius $k_{h}$ is taken to obtain the twodimensional energy spectrum in each plane $E\left(k_{h}, z\right)$. Finally all of these horizontal spectra are averaged over the vertical coordinate to obtain the two-dimensional horizontal energy spectrum $E\left(k_{h}\right)=\left\langle E\left(k_{h}\right)\right\rangle_{z}$. Figure 17 shows the horizontal energy spectra as a function of the horizontal wavenumber at different stages of the experiment. As soon as the flow becomes quasi-horizontal and for the duration of the experiment, the energy remains confined to the large scales. The slope in the high-wavenumber region is not constant and decreases with time from about 3.8 to 2. In all the experiments we observe a convergence to this slope of approximately 2. 


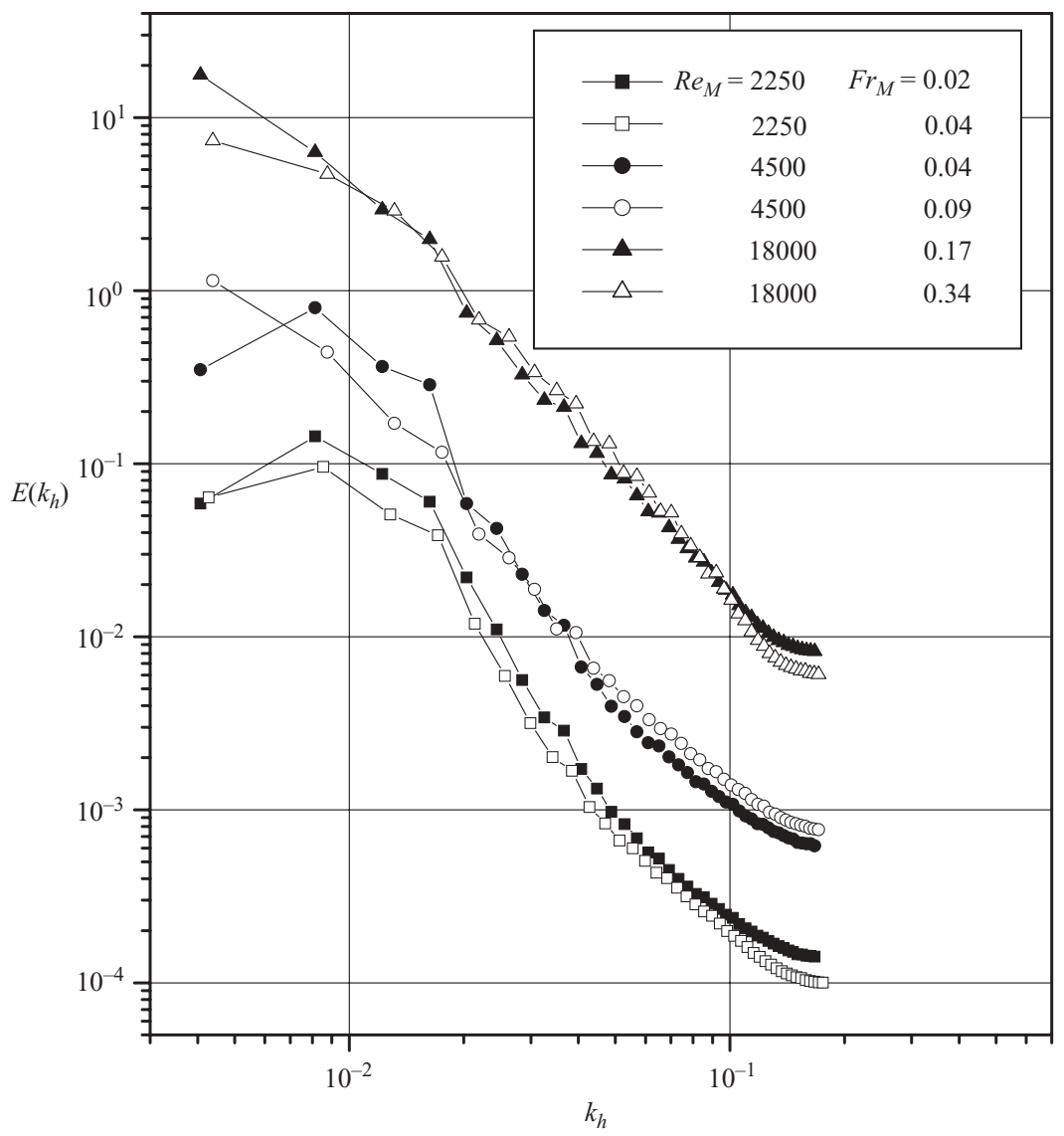

FIgURE 18. Two-dimensional energy spectra for various experiments at $t^{*} \sim 20$.

The effect of the experimental parameters $F_{M}$ and $R e_{M}$ is shown in figure 18 where the two-dimensional energy spectra for various experiments at the same advective time $t^{*} \sim 20$ are plotted. As expected, the level of the spectra increases with $U$ as the flow is more energetic at all scales, but the remarkable fact is that very little dependence of the horizontal energy spectra on the stratification is seen at all measured scales.

Figure 19 shows, at two different times $\left(t^{*}=20\right.$ and 80$)$, the horizontal energy spectra normalized with $U^{2}$ versus the horizontal wavenumber normalized with $M^{-1}$. In all cases, the spectra collapse relatively well onto a single curve. This similarity, obtained at all scales in the inertial range, indicates clearly that the dynamics of the quasi-horizontal motion does not depend on the stratification or the Reynolds number. Moreover, the slope of the spectra, as already observed in figure 17, decreases with time and seems to converge toward a -2 slope. Different theoretical models predict such $k_{h}^{-2}$ spectra, for instance Herring (1988) and Yeung \& Zhou (1998) in an equivalent rotating turbulence problem. They are obtained as well in weakly interactive wave theories (Staquet \& Sommeria 2002). However, all these models predict a dependence of the spectral energy on the stratification $N$ unlike what is observed in our experiments. 

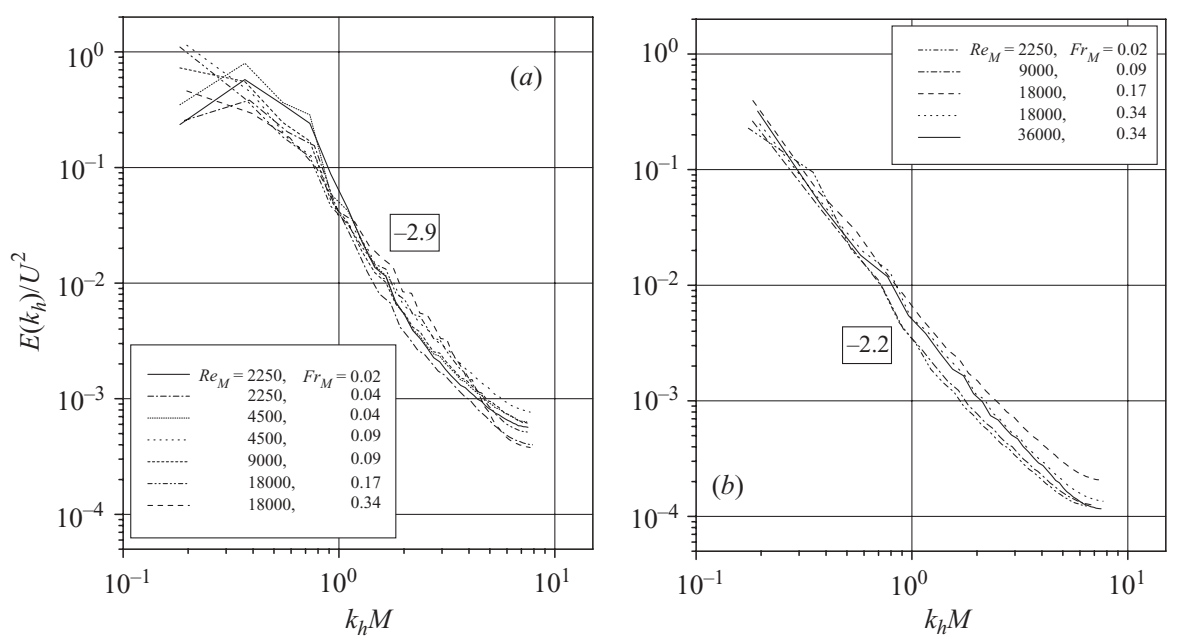

FIGURE 19. Normalized kinetic energy versus normalized horizontal wavenumber at (a) $t^{*} \sim 20$ and $(b) t^{*} \sim 80$.

\subsection{Vertical spectra}

Figure 20 shows the vertical spectra of kinetic energy for different experiments (ba, $\mathrm{bb}, \mathrm{bd}, \mathrm{ia}, \mathrm{ib}, \mathrm{id})$ at $t^{*} \sim 30$. Like the horizontal energy spectra, the vertical spectra, at almost all scales, are not affected by stratification.

Figure 21 shows the result of scaling the vertical spectra of kinetic energy with the Kolmogorov scale. The vertical wavenumber is normalized with $\eta=\left(\epsilon / \nu^{3}\right)^{1 / 4}$ and the energy with $v^{2} \eta$. The measurement of $\epsilon$ is described in $\S 6$. The curves in figure 21 are a subset of the entire range of $t^{*}$ of experiment bd. The collapse of the scaled spectra is surprisingly good over most of the wavenumber range and also for the low-wavenumber region. This similarity of the spectra with time was not observed in the horizontal spectra where the slope was decreasing with time. The slope of these vertical spectra remains constant with time and close to -3 . It must be noted that these $k_{z}^{-3}$ spectra are not associated with a saturation of internal waves as the energetic contribution of the waves is very weak. $E\left(k_{z}\right)$ definitely represents the vertical spectra associated with the vortical velocity field. The Kolmogorov scaling is not only successful for time evolution but also over the range of stratification and initial Reynolds numbers. Figure 22 shows the vertical energy spectra, normalized by the Kolmogorov scales, for various experiments (ba, bb, bc, bd, ia, ib, id), at $t^{*}=40$. With this scaling the collapse of all the spectra is once again surprisingly good over most of the wavenumber range. The deviation observed at the largest wavenumbers is partially due to the noise introduced by the PIV measurement, which depends on the physical scale of the motion.

This similarity and the constant $k_{z}^{-3}$ slope observed for the vertical spectra of kinetic energy confirm that the flow cannot be considered as a superposition of completely independent layers of two-dimensional turbulence. If this were the case, the vertical spectra would tend to resemble a white noise spectrum.

In addition, as the vertical shearing is responsible for most of the dissipation, we can estimate the dissipation spectrum from the vertical spectrum:

$$
\epsilon\left(k_{z}\right)=k_{z}^{2} E\left(k_{z}\right) \text {. }
$$




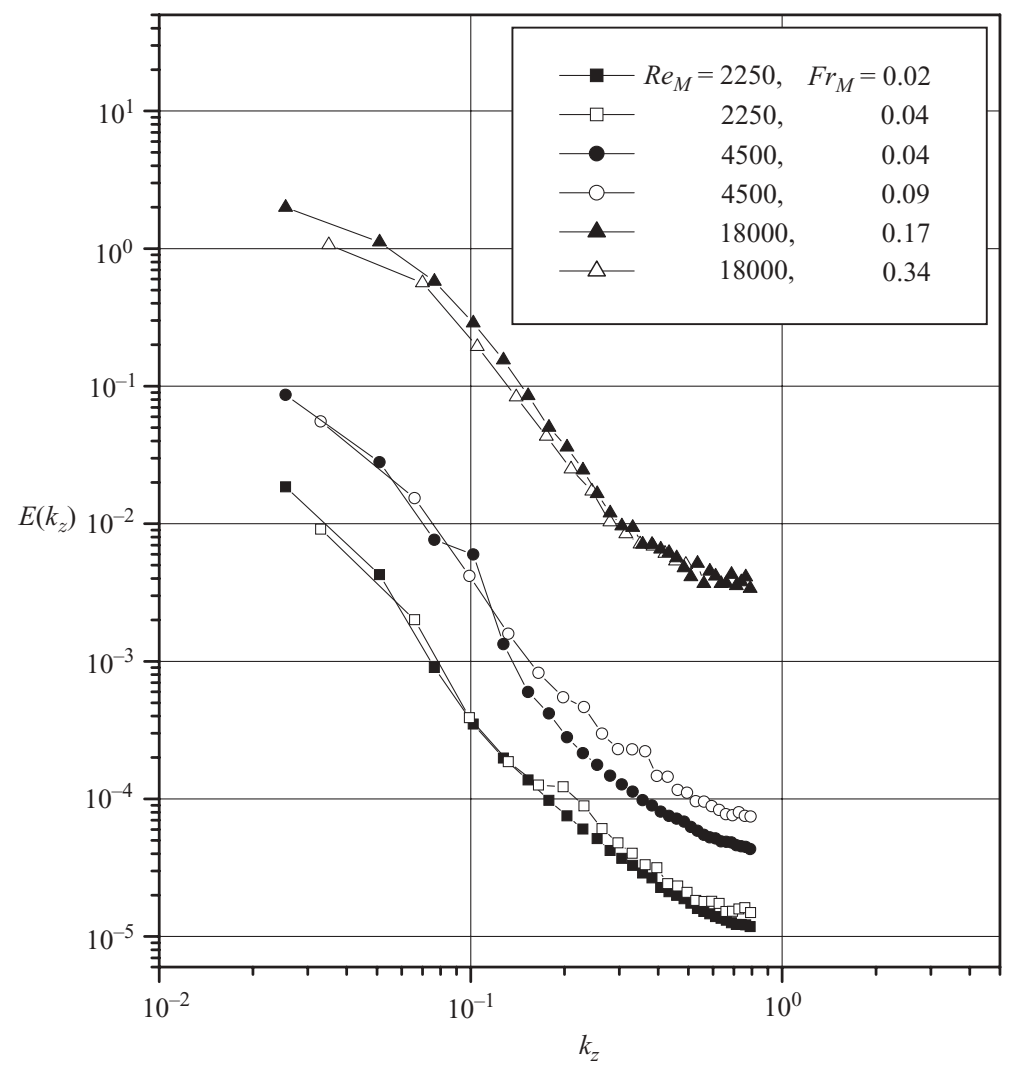

FIGURE 20. Kinetic energy versus vertical wavenumber at $t^{*} \sim 30$ for different experiments.

The dissipation associated with each scale $k_{z}$ is then given by integration of $\epsilon\left(k_{z}\right)$ from $k_{z}$ to $2 k_{z}$. The $k_{z}^{-3}$ slope of the vertical spectra leads thus to a $k_{z}^{-1}$ dissipation spectra without a distinguishable preferential dissipation scale. All length scales contribute almost equally to the dissipation of turbulent kinetic energy.

\section{Wave-vortex analysis}

In the late stages of freely decaying turbulence in strong stratification $\mathrm{Fr}_{h} \ll 1$ and the curvature of the isopycnes is small. In this limit, we can quantify the relative importance of the internal waves by using the following decomposition of the velocity field:

$$
\hat{u}_{i}=\hat{\phi}^{1} e_{i}^{1}+\hat{\phi}^{2} e_{i}^{2}
$$

where the unit vectors $\boldsymbol{e}^{1}$ and $\boldsymbol{e}^{2}$ are defined as follows. Since $\boldsymbol{u}$ is perpendicular to $\boldsymbol{k}$ because of the continuity equation $(\nabla \cdot \boldsymbol{u}=0)$, a local frame of reference is defined in Fourier space, made up of the wavevector $\boldsymbol{k}$ and of two unit vectors lying in a plane $\Pi$ perpendicular to $\boldsymbol{k}$. One of the two vectors, $\boldsymbol{e}^{1}(\boldsymbol{k})$, is taken horizontally; $\boldsymbol{e}^{2}(\boldsymbol{k})$ lies along the intersection of $\Pi$ and a vertical plane. The last vector $\boldsymbol{e}^{3}(\boldsymbol{k})$ completes the frame. This frame is usually referenced as the Craya-Herring frame (Craya 1958). It was also proposed by Chandrasekar (1961) who introduced the equivalent poloidal, 


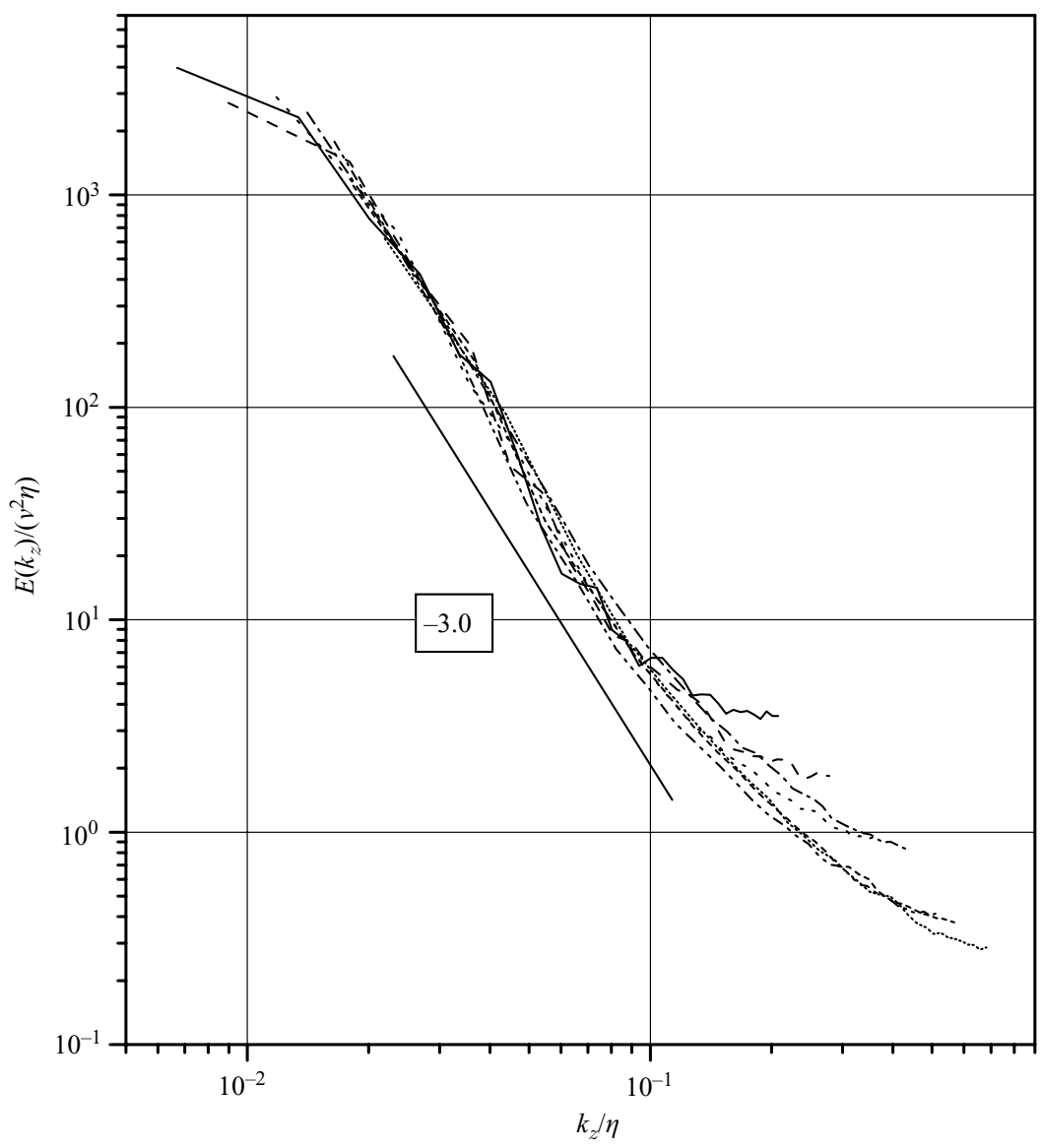

FIGURE 21. Vertical spectra of kinetic energy non-dimensionalized by the Kolmogorov scale at $t^{*}=40,70,100,160,210,275,350 . R e_{M}=18000, F_{M}=0.17$.

toroidal decomposition. The units vectors are then defined by

$$
\begin{gathered}
\boldsymbol{e}^{1}(\boldsymbol{k})=(\boldsymbol{k} \times \boldsymbol{n}) /|\boldsymbol{k} \times \boldsymbol{n}|, \\
\boldsymbol{e}^{2}(\boldsymbol{k})=\left(\boldsymbol{k} \times \boldsymbol{e}^{1}\right) / \mid \boldsymbol{k} \times \boldsymbol{e}^{1}, \\
\boldsymbol{e}^{3}(\boldsymbol{k})=\boldsymbol{k} / k,
\end{gathered}
$$

where $\boldsymbol{n}$ is the vertical unit vector.

This decomposition can also be formulated in physical space. Splitting the velocity vector $\boldsymbol{u}$ into its horizontal and vertical parts and applying the Helmholtz decomposition to the former components leads to

$$
\boldsymbol{u}=\nabla_{h} \times \psi \boldsymbol{n}+\overline{\boldsymbol{u}}_{h}+\nabla_{h} \zeta+u_{z} \boldsymbol{n}
$$

where $\nabla_{h}$ represents the gradient operator in a horizontal plane and $\overline{\boldsymbol{u}}_{h}$ the vertically sheared horizontal flows (VSHF) associated with the modes with $k_{h}=\left(k_{x}^{2}+k_{y}^{2}\right)^{1 / 2}=0$. It can be easily shown that, for an incompressible flow, the Fourier transforms of $\psi$ and $\zeta$, respectively $\hat{\psi}$ and $\hat{\zeta}$, are related to $\hat{\phi}^{1}$ and $\hat{\phi}^{2}$ by the relations

$$
\hat{\phi}^{1}=\mathrm{i} k_{h} \hat{\psi}, \quad \hat{\phi}^{2}=\mathrm{i} \frac{k_{h} k}{k_{z}} \hat{\zeta}
$$




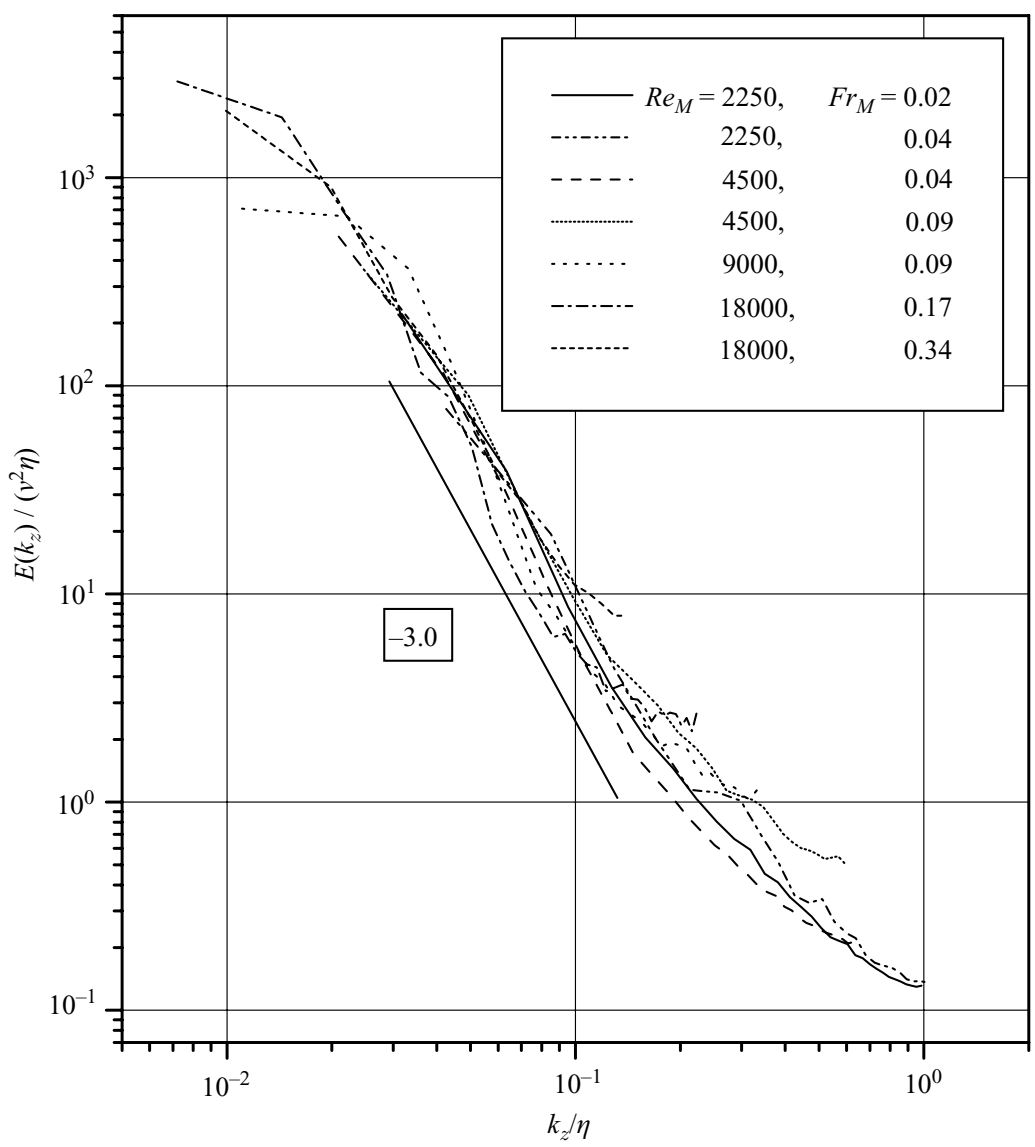

FIGURE 22. Vertical spectra of kinetic energy non-dimensionalized by the Kolmogorov scale at $t^{*}=40$ for different experiments.

Riley et al. (1981) shown that for a stably stratified fluid, in the linear limit $\nabla_{h} \times \psi \boldsymbol{n}$ defines the non-propagating or vortex part of the flow which contains all vertical vorticity while the two last terms of (5.5) define the wave part and contain all of the vertical velocity. The VSHF modes are divergent free, do not contain vertical vorticity and have kinetic energy only.

In our study only the horizontal components of the velocity fields are measured. We thus take the horizontal part of (5.5). The corresponding scalars $\psi$ and $\zeta$ are uniquely determined by taking respectively the curl and the horizontal divergence of $\boldsymbol{u}_{h}$ leading to

$$
\begin{aligned}
-\omega_{z} & =\nabla^{2} \psi, \\
\nabla_{h} \cdot \boldsymbol{u}_{h} & =\nabla_{h}^{2} \zeta,
\end{aligned}
$$

where $\nabla_{h}^{2}$ denotes the Laplacian operator in a horizontal plane.

By solving numerically these two Poisson equations (5.7) and (5.8) we separate the horizontal flow into its vortical and horizontal wave parts. The vortex part of the flow is purely horizontal while the wave part contains all the vertical velocity. As the VSHF modes contain horizontal kinetic energy and may be associated with vortical structures 


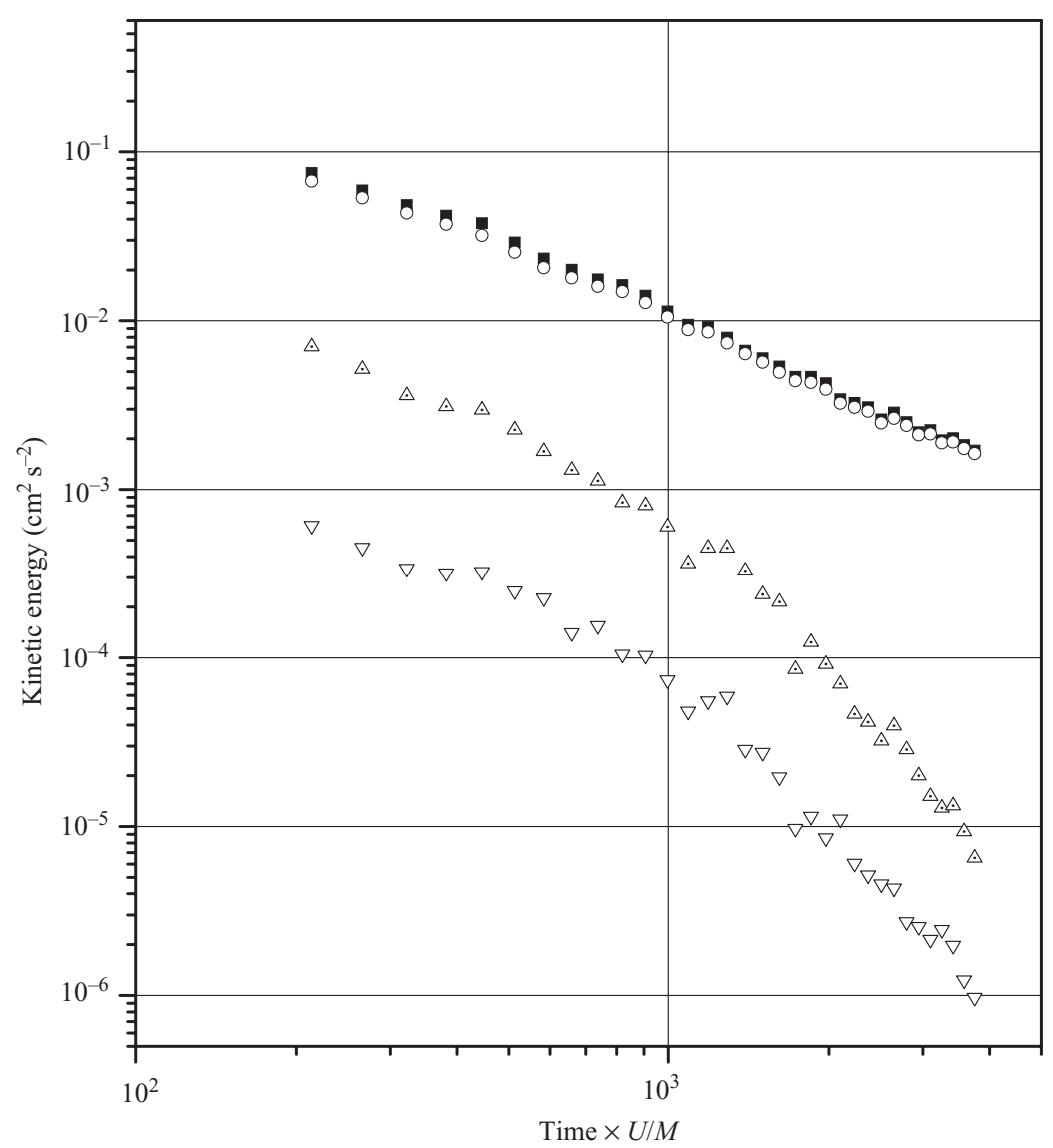

FIGURE 23. Evolution of the total kinetic energy (black squares), vortex horizontal kinetic energy (circles), wave kinetic energy (upward triangles) and vertical kinetic energy (downward triangles). $\operatorname{Re}_{M}=9000, \mathrm{Fr}_{M}=0.08, N=0.52$.

much larger than the measurement area, they are included in the vortical part of our decomposition.

The total kinetic energy, its vortical and wave contributions and the vertical kinetic energy are plotted versus time in figure 23. The first measurement was made for $N t \sim 100$. At this time the waves generated during the collapse of the initial turbulent patches have already been radiated away. Therefore, only the wave field directly associated with the low-Froude-number turbulence remains. These curves show the small contribution of the waves to the horizontal kinetic energy $(10 \%$ at $N t \sim 100$ in this case). The decay rate of the wave part is also faster than the vortex part, indicating that the energetic domination of the vortex mode increases with time.

This relative contribution of the wave part is directly related to the importance of stratification. Figure 24 shows how this ratio increases with the Froude number $\mathrm{Fr}_{M}$ (at a fixed time $N t \sim 125$ ). The contribution of the wave part increases with $F r_{M}$ but in all cases this contribution remains weak and, as just discussed, decreases with time.

The wave kinetic energy can itself be decomposed into a contribution of the horizontal and vertical velocity components (figure 23), and the latter contribution is small (less than 10\%). Accordingly, the wave field is dominated by wavevectors $\boldsymbol{k}$ close to the vertical, $k_{z} \gg k_{h}$. Their frequency $\omega$, given by the dispersion relation 


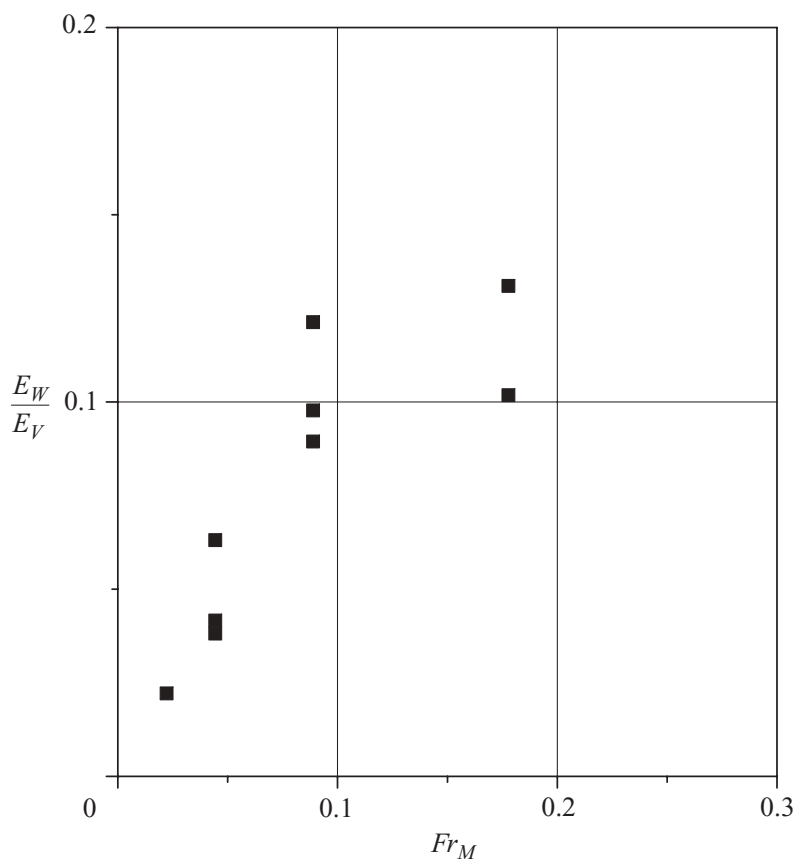

FIGURE 24. Ratio of the horizontal kinetic energy of the wave part to the total horizontal kinetic energy versus Froude number. $N t \sim 125$. Experiments aa, ab, ac, ba, bc, bd, ia, ib.

$\omega^{2}=N^{2} \boldsymbol{k}_{h}^{2}\left(\boldsymbol{k}_{h}^{2}+\boldsymbol{k}_{z}^{2}\right)^{-1}$, is therefore much smaller than $N$. We can estimate that this is close to the advective frequency $u / L_{h}=N / F r \ll N$, allowing strong interactions between the vortical and wave modes.

\section{Mechanisms of energy dissipation}

\subsection{Anisotropy of the dissipation}

The organization of the flow into layers of eddies separated by intense and highly dissipative sheets of horizontal vorticity leads to a strong anisotropy of the dissipation: the vertical shear of the horizontal velocity components dominates. We here analyse in more detail the contributions of the different shear directions to energy dissipation.

The dissipation rate of the turbulent kinetic energy $E=\frac{1}{2}\left(u_{1}^{2}+u_{2}^{2}+u_{3}^{2}\right)$ is given locally by

where

$$
\epsilon=2 v\left\langle s_{i j} s_{i j}\right\rangle
$$

$$
s_{i j}=\frac{1}{2}\left(\frac{\partial u_{i}}{\partial x_{j}}+\frac{\partial u_{j}}{\partial x_{i}}\right), \quad i, j=1,2,3 .
$$

As the three-dimensional SCIV technique provides the horizontal components of the velocity field in a volume, all components of the deformation tensor $s_{i j}$ are measured except the horizontal derivatives of the vertical velocity. The vertical derivative of the vertical velocity is estimated from the continuity equation.

Following the analysis of Fincham et al. (1996), the dissipation owing to gradients in a plane normal to the unit vector $\boldsymbol{e}_{k}$ is given by

$$
\epsilon_{k}=2 v\left\langle s_{i j} s_{i j}\right\rangle, \quad i, j \neq k .
$$




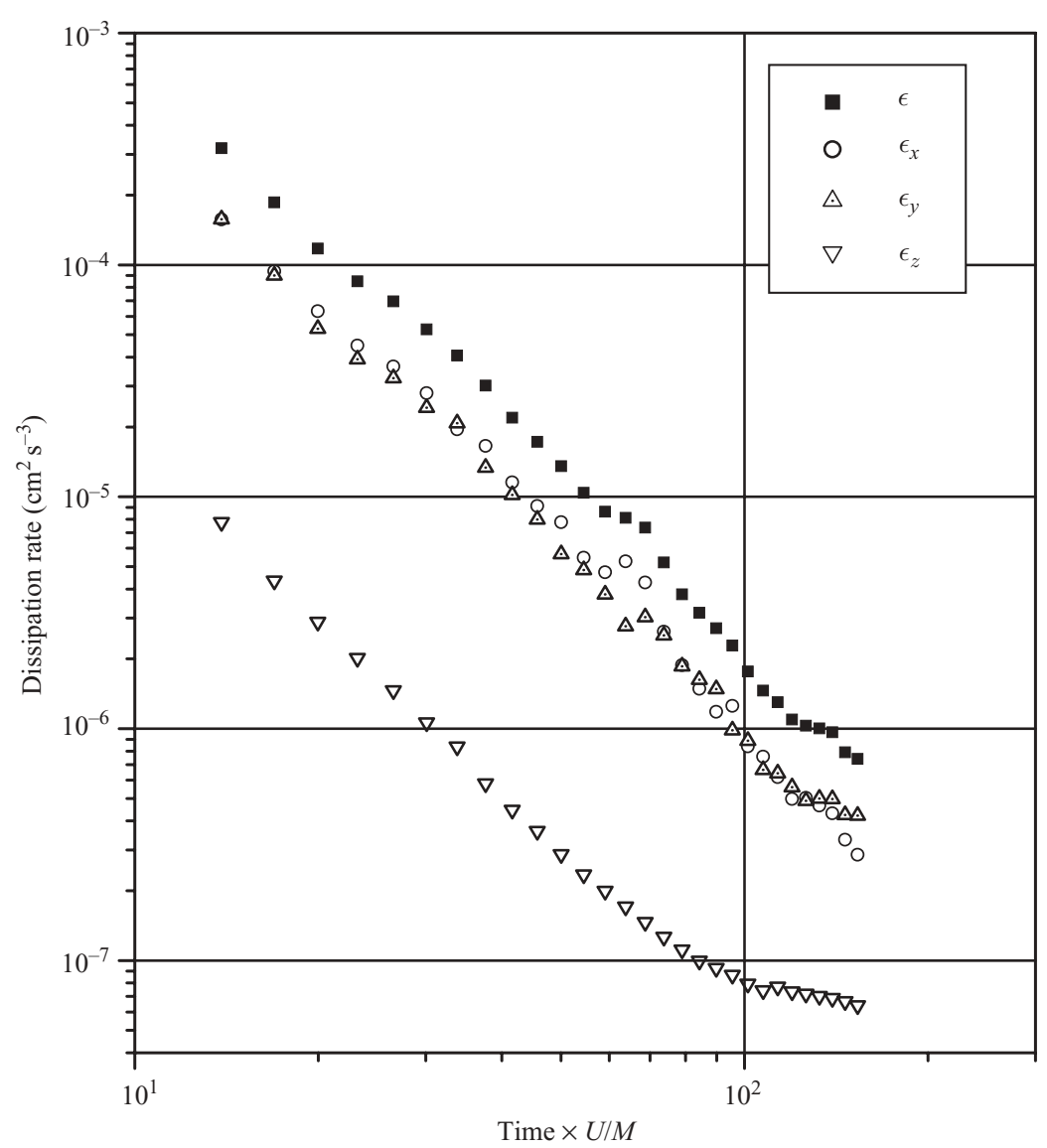

FIgURE 25. Contributions of $\epsilon_{x}, \epsilon_{y}, \epsilon_{z}$, to the total dissipation of horizontal kinetic energy.

$$
\operatorname{Re}_{M}=9000, \mathrm{Fr}_{M}=0.09 \text {. }
$$

The contribution of the derivatives in the three directions $(x, y, z) \equiv\left(x_{1}, x_{2}, x_{3}\right)$ of the velocity components $\left(u_{x}, u_{y}, u_{z}\right) \equiv\left(u_{1}, u_{2}, u_{3}\right)$ are given by

$$
\begin{aligned}
\epsilon_{x} & =v\left\langle 2\left(\frac{\partial u_{y}}{\partial y}\right)^{2}+2\left(\frac{\partial u_{z}}{\partial z}\right)^{2}+\left(\frac{\partial u_{z}}{\partial y}\right)^{2}+\left(\frac{\partial u_{y}}{\partial z}\right)^{2}+2\left(\frac{\partial u_{y}}{\partial z} \frac{\partial u_{z}}{\partial y}\right)\right\rangle, \\
\epsilon_{y} & =v\left\langle 2\left(\frac{\partial u_{x}}{\partial x}\right)^{2}+2\left(\frac{\partial u_{z}}{\partial z}\right)^{2}+\left(\frac{\partial u_{z}}{\partial x}\right)^{2}+\left(\frac{\partial u_{x}}{\partial z}\right)^{2}+2\left(\frac{\partial u_{x}}{\partial z} \frac{\partial u_{z}}{\partial x}\right)\right\rangle, \\
\epsilon_{z} & =v\left\langle 2\left(\frac{\partial u_{y}}{\partial y}\right)^{2}+2\left(\frac{\partial u_{x}}{\partial x}\right)^{2}+\left(\frac{\partial u_{x}}{\partial y}\right)^{2}+\left(\frac{\partial u_{y}}{\partial x}\right)^{2}+2\left(\frac{\partial u_{y}}{\partial x} \frac{\partial u_{x}}{\partial y}\right)\right\rangle .
\end{aligned}
$$

The total turbulent viscous dissipation can then be written as

$$
\epsilon=\epsilon_{x}+\epsilon_{y}+\epsilon_{z}-2 \nu\left\langle\left(\frac{\partial u_{x}}{\partial x}\right)^{2}+\left(\frac{\partial u_{y}}{\partial y}\right)^{2}+\left(\frac{\partial u_{z}}{\partial z}\right)^{2}\right\rangle .
$$

We neglect in these formula the unmeasured contributions of $\partial u_{z} / \partial x$ and $\partial u_{z} / \partial y$, which involve the weak $u_{z}$ component combined with weak derivatives in the horizontal directions. The vertical derivative of $u_{z}$ is obtained from the continuity equation $\partial u_{z} / \partial z=-\partial u_{x} / \partial x-\partial u_{y} / \partial y$.

In figure 25, we examine the contribution of $\epsilon_{x}, \epsilon_{y}$ and $\epsilon_{z}$ to the total dissipation. The isotropy of the flow in the horizontal direction is satisfied as the contributions of $\epsilon_{x}$ and 


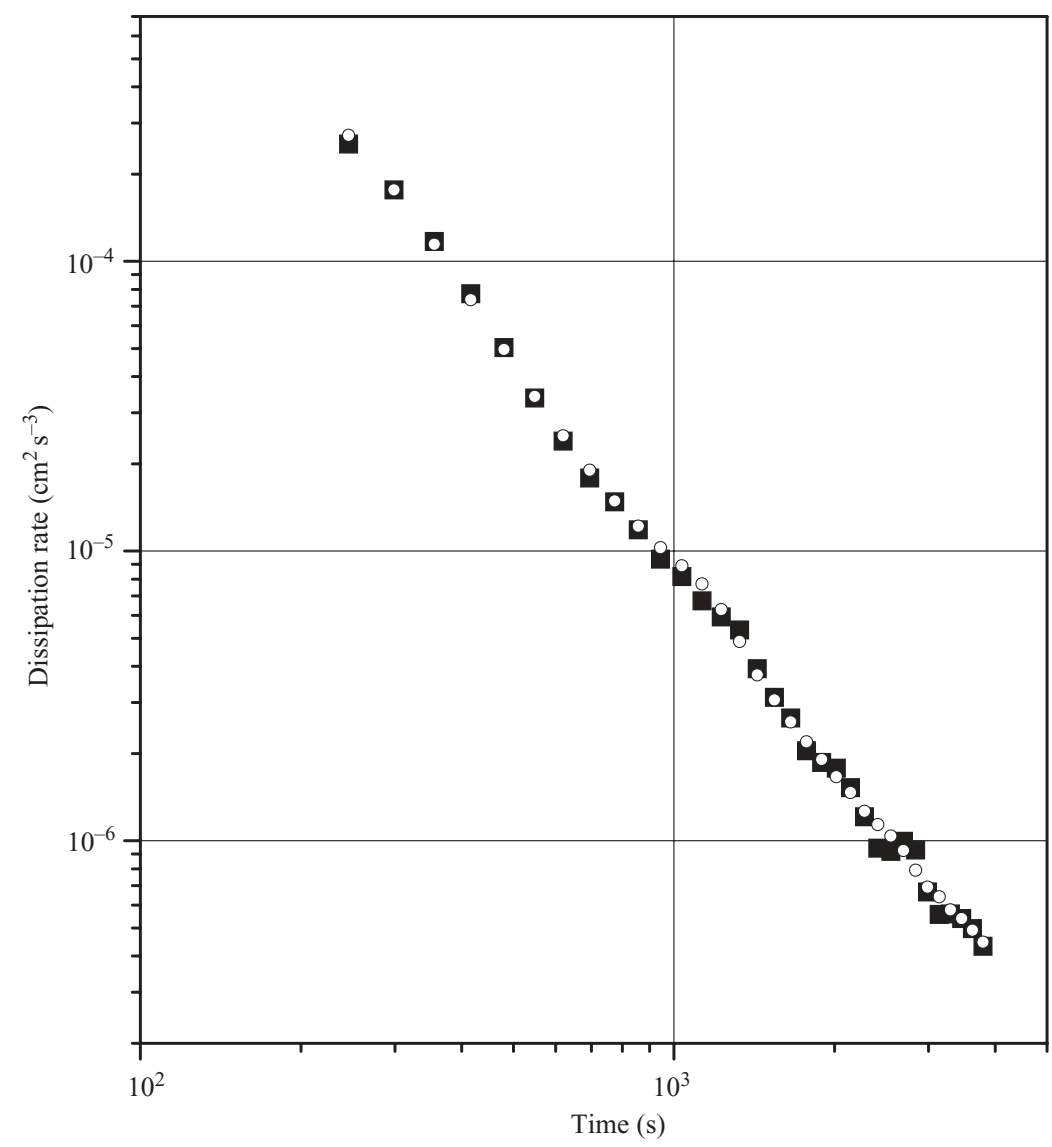

FigURE 26. Evolution of the dissipation rate of horizontal kinetic energy, estimated from $-\mathrm{d} E / \mathrm{d} t$ (black squares) and from $2 v s_{i j} s_{i j}$ (open circles). $R e_{M}=4500, F r_{M}=0.04$.

$\epsilon_{y}$ remain the same in time while a strong anisotropy between vertical and horizontal directions is evident. It is seen clearly that the dissipation is dominated by the vertical gradients which contribute, in this case, more than $90 \%$. The vertical shearing dominates the dissipation and is responsible for the quasi-totality of the energy decay. Therefore, assuming horizontal isotropy, $\epsilon$ can be estimated by $\epsilon \simeq 2 \epsilon_{y}=2 v\left\langle\left(\partial u_{x} / \partial z\right)^{2}\right\rangle$ confirming our previous estimate (3.8).

\subsection{The viscous dissipation rate}

The reliability of the estimation of $\epsilon$ through (6.1) where the contributions of $\partial u_{z} / \partial x$ and $\partial u_{z} / \partial y$ are not taken into account, is shown in figure 26 . The very good agreement between the evaluation from the time derivative of the turbulent kinetic energy $-\mathrm{d} E / \mathrm{d} t$ and from $2 v s_{i j} s_{i j}$ shows that the measurement technique permits an accurate resolution of the dissipation scales. This agreement also confirms the weak contribution of the derivative of the vertical velocity to the dissipation. There is also a possible exchange between kinetic and potential energies, and potential energy dissipation by salt diffusion. The latter effects are also negligible in the low-Froude-number regime because of the weak internal wave component and the low salt diffusivity.

The dissipation rate of kinetic energy for different experiments is plotted versus time in figure 27. All the curves collapse well when $\epsilon$ is normalized by $U^{3} / M$, to the law $\epsilon^{*} \simeq 0.5 t^{*-2.3}$. This is again in good agreement with the law for $\mathrm{d} E / \mathrm{d} t$ obtained from the measured energy (3.1). 


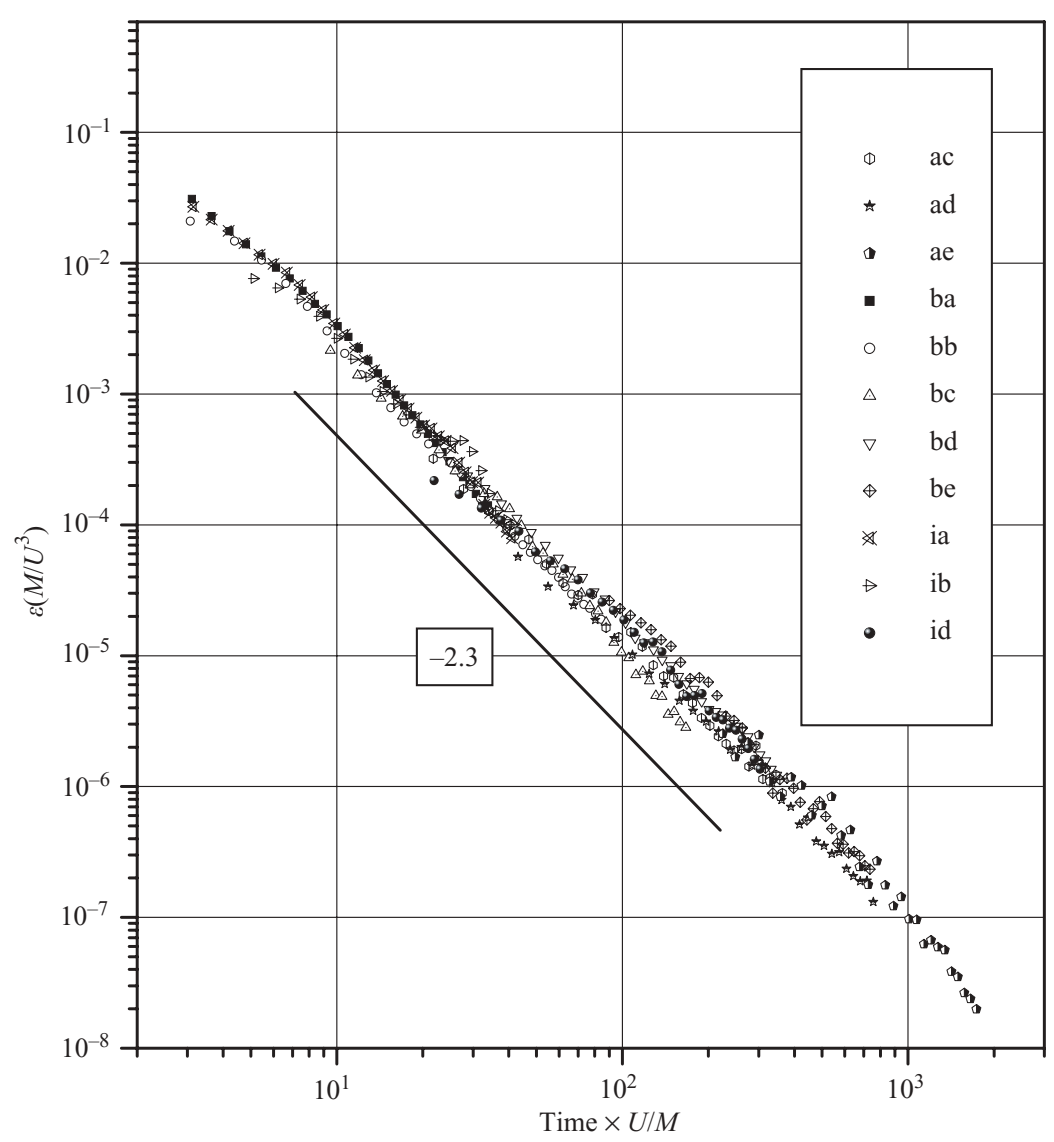

FIGURE 27. Decay of the normalized dissipation rate of the kinetic energy. Experiments are listed in table 1.

As previously observed, the decay law is the same as in the isotropic homogeneous case, although the turbulence is here highly anisotropic. Moreover, the rate of energy dissipation satisfies the usual Kolmogorov estimate $\epsilon \sim u^{3} / L_{h}$, with the horizontal integral scale $L_{h}$. Indeed from (3.1) and (3.3), we obtain

$$
\epsilon \simeq c u^{3} / L_{h} \quad \text { with } \quad c=0.6 t^{*-0.15},
$$

which is nearly constant. It is about half the corresponding value in isotropic turbulence, which means that the energy transfer process is about half as efficient as in the usual Kolmogorov cascade. We can alternatively estimate $u / L_{h}$ from the vertical vorticity $\left\langle\omega_{z}^{2}\right\rangle$, shown in figure 14 . It yields $u / L_{h} \sim t^{*-1}$, so that $(\mathrm{d} E / \mathrm{d} t) / E \sim-u / L_{h}$. This result suggests that the dynamics is controlled by the horizontal advection, which tends to decorrelate the layers. This energy transfer from horizontal to vertical wave vectors is balanced by viscous dissipation due to the resulting vertical shear.

\section{Conclusion}

Decaying stratified grid-generated turbulence has been investigated using a scanning particle image velocimetry technique providing time-resolved velocity fields in a volume. We considered the regime of quasi-horizontal motion obtained after the collapse of the three-dimensional stratified turbulence. 
In this regime, we observe the evolution of the flow into a layered field with strong vertical shear. The generation of such horizontal layers shows good agreement with the results obtained in the numerical simulations of Herring \& Metais (1989) or in the experiments of Fincham et al. (1996). We find the layering scale to be independent of the stratification and in particular it does not exhibit any proportionality with $U / N$.

While the horizontal motion dominates, the vertical shear leads to strong energy dissipation and prevents stratified turbulence from exhibiting the characteristics of two-dimensional turbulence. This anisotropy of the dissipation process was quantified using a direct measurement of the relative contribution of all the gradients to the dissipation rate. Vertical shearing appears to be responsible for the quasi-totality of the energy decay, increasingly so for higher Reynolds numbers.

The present set of experiments demonstrates some important properties of decaying stratified turbulence, once the quasi horizontal motion regime is established. We found in this regime a self-similar temporal evolution of the energy decay and of the length scales. The kinetic energy decay, remarkably, exhibits the same scaling form as threedimensional isotropic homogeneous turbulence with a scaling exponent close to -1.3 , even though the energy dissipation mechanisms are radically different. This value is also close to the one found in the early stage (before the collapse) of decaying stratified turbulence. The scaling law obtained for the evolution of the vertical scale, $R e_{M}^{-0.5} t^{* 0.5}$, is consistent with the model of viscous growth proposed in this paper.

Moreover, very little influence of the initial conditions and in particular of the stratification was observed in either the dynamics or the measured values of the kinetic energy and the horizontal and vertical length scales. Our results can therefore be considered as general properties of stratified turbulence independent of the generation mechanism. Furthermore, as pointed out by Riley et al. (1981), whatever the initial Froude number, the turbulence is completely dominated by stratification after a few Brunt-Väisälä periods. Stratification plays a role in the early stages of the turbulence evolution by inhibiting the vertical fluxes and provoking its collapse. But, after the collapse, with the establishment of the quasi-horizontal motion, the dynamics of the flow is no longer controlled by stratification and does not depend on it. In this regime $(F r \ll 1)$ a self-similarity in the limit of $F r \rightarrow 0$ is suggested by the experiments.

Nevertheless, the dynamics of the turbulence cannot be represented by completely independent quasi-horizontal flows. Indeed, we showed that the layers are strongly coupled by viscosity. A balance between horizontal advection, which tends to vertically decorrelate the flow, and vertical diffusion is observed. The basic dynamics of energy decay is therefore a transfer from large horizontal to smaller vertical scales, where viscous dissipation takes place. As a consequence of this viscous coupling, the vertical scales are found to be governed by viscosity and to grow according to a diffusive process that is not influenced by their initial behaviour. Note that all the vertical scales contribute equally to this dissipation because of the $k_{z}^{-3}$ energy spectrum.

Extrapolating these results to very high Reynolds numbers, we find that the vertical shear scales as $\omega_{h} \sim R e^{0.5}$. For high $R e$, the condition for low gradient Richardson number $R i=N^{2} / \omega_{h}^{2} \sim N^{2} R e^{-1}$ will clearly be reached, leading to local shear instability. This will lead to the generation of small-scale turbulence that could act as an eddy diffusivity for the larger scales, so our results could apply with a Reynolds number based on this eddy diffusivity. In particular the scaling laws for the velocity and length scales will be the same. It is expected that this high-Reynolds-number case would not be easily realized in freely decaying laboratory experiments, as the three dimensional process of turbulent collapse used to generate the pancake vortices dissipates sufficient energy to leave the resulting quasi-horizontal flow in the stable regime. It should be 
possible in a forced experiment to maintain a sufficiently large Reynolds number to find zones with Richardson numbers smaller than $1 / 4$.

These laboratory experiments would not have been possible without the support of Dr Henri Didelle and Mr Rene Carcel.

\section{REFERENCES}

Billant, P. \& Chomaz, J. M. $2000 a$ Experimental evidence for a new instability of a vertical columnar vortex pair in a strongly stratified fluid. J. Fluid Mech. 418, 167-188.

Billant, P. \& Chomaz, J. M. $2000 b$ Theoretical analysis of the zigzag instability of a vertical columnar vortex pair in a strongly stratified fluid. J. Fluid Mech. 419, 29-63.

Bonnier, M., Eiff, O. \& Bonneton, P. 2000 On the density structure of far-wake vortices in a stratified fluid. Dyn. Atmos. Oceans 31, 117-137.

Britter, R. E., Hunt, J. C. R., Marsh, G. L. \& Snyder, W. H. 1983 The effect of stable stratification on turbulent diffusion and the decay of grid turbulence. J. Fluid Mech. 127, 27-44.

Chandrasekar, S. 1961 Hydrodynamic and Hydromagnetic Stability. Clarendon.

Comte-Bellot, G. \& Corrsin, S. 1966 The use of a contraction to improve the isotropy of a grid generated turbulence. J. Fluid Mech. 25, 657-682.

CrayA, A. 1958 Contribution a l'analyse de la turbulence associee à des vitesse moyenne. Tech. Rep. 345, Ministère de l'Air.

Dickey, T. D. \& Mellor, G. L. 1980 Decaying turbulence in neutral and stratified fluids. J. Fluid Mech. 99, 13-31.

FIncham, A. M. 1998 3D measurement of vortex structures in stratified fluid flows. In IUTAM Symp. On Simulation and Identification of Organized Structures in Flows (ed. E. J. H. J. N. Soerensen \& N. Aubry), pp. 273-287.

Fincham, A. M. \& Delerce, G. 2000 Advanced optimization of correlation imaging velocimetry algorithms. Exps. Fluids. Suppl. 29, S13-S22.

Fincham, A. M., Maxworthy, T. \& Spedding, G. R. 1996 Energy dissipation and vortex structure in freely decaying stratified grid turbulence. Dyn. Atmos. Oceans 23, 155-169.

Fincham, A. M. \& SPEDding, G. R. 1997 Low cost, high resolution DPIV for measurement of turbulent fluid flow. Exps. Fluids 23, 449-462.

Flor, J. B., van Heisst, G. J. F. \& Delfos, R. 1995 Decay of dipolar vortex structures in a stratified fluid. Phys. Fluids 7, 374-383.

Herring, J. R. 1988 The inverse cascade range of quasi-geostrophic turbulence. Met. Atmos. Phys. 38, $106-115$.

Herring, J. R. \& Metais, O. 1989 Numerical simulation in forced stably stratified turbulence. J. Fluid Mech. 202, 97-115.

Hinze, J. 1975 Turbulence, 2nd edn. McGraw-Hill.

Hopfinger, E. J. 1987 Turbulence in stratified fluid: a review. J. Geophys. Res. 92, 5287-5303.

Itsweire, E. C., Helland, K. N. \& Van AtTa, C. W. 1986 The evolution of grid generated turbulence in a stably stratified fluid. J. Fluid Mech. 162, 299-338.

Kimura, Y. \& Herring, J. R. 1996 Diffusion in stably stratified turbulence. J. Fluid Mech. 328, 253-269.

Lienhard, J. H. \& VAn Atta, C. W. 1990 The decay of turbulence in thermally stratified flow. J. Fluid Mech. 210, 57-112.

Lin, J. T. \& PAO, Y. H. 1979 Wakes in stratified fluids. Annu. Rev. Fluid Mech. 11, 317-338.

MajdA, A. J. \& Grote, M. J. 1997 Model dynamics and vertical collapse in decaying strongly stratified flows. Phys. Fluids 9, 2932-2940.

Metais, O. \& Herring, J. R. 1989 Numerical simulations of freely evolving turbulence in stably stratified fluids. J. Fluid Mech. 202, 117-148.

PAIHUA, L. 1978 Quelques méthodes numériques pour le calcul de fonctions splines à une et plusieurs variables. PhD thesis, Universite de Grenoble.

Park, Y. G., Whitehead, J. A. \& Gnanadeskian, A. 1994 Turbulent mixing in stratified fluids: layer formation and energetics. J. Fluid Mech. 279, 279-311. 
Riley, J. J. \& Lelong, M. P. 2000 Fluid motions in the presence of strong stable stratification. Annu. Rev. Fluid Mech. 32, 613-657.

Riley, J. J., Metcalfe, R. W. \& Weissman, M. A. 1981 Direct numerical simulations of homogeneous turbulence in density-stratified fluids. In Proc. Conf. on Nonlinear Properties of Internal Waves (ed. J. B. West), pp. 79-112. American Institute of Physics.

De RooiJ, F., Linden, P. F. \& Dalziel, S. B. 1999 Experimental investigations of quasi-twodimensional vortices in a stratified fluid with source-sink forcing. J. Fluid Mech. 383, 249-283.

Spedding, G. R. \& Rignot, E. J. M. 1993 Performance analysis and application of grid interpolation techniques for fluid flows. Exps. Fluids 15, 417-430.

Staquet, C. \& Godeferd, F. S. 1998 Statistical modelling and direct numerical simulations of decaying stably stratified turbulence. Part 1: Flow energetics. J. Fluid Mech. 360, 295-340.

Staquet, C. \& Sommeria, J. 2002 Internal gravity waves, from instability to turbulence. Annu. Rev. Fluid Mech. 34, 559-593.

Stillinger, D. C., Helland, K. N. \& Van Atta, C. W. 1983 Experiments on the transition of homogeneous turbulence to internal waves in a stratified fluid. J. Fluid Mech. 131, 91-122.

Voropayev, S. I., Afanasyez, Y. D. \& van Heisst, G. J. F. 1995 Two-dimensional flows with zero net momentum: evolution of vortex quadripoles and oscillating-grid turbulence. J. Fluid Mech. 282, 21-44.

WARHAFt, Z. \& LumLey, J. L. 1978 Experimental study of decay temperature fluctuations in grid generated turbulence. J. Fluid Mech. 88, 659-684.

YAP, C. T. \& VAN ATTA, C. W. 1993 Experimental studies of the development of quasi-twodimensional turbulence in stably stratified fluid. Dyn. Atmos. Oceans 19, 289-323.

Yeung, P. K. \& Zhou, Y. 1998 Numerical study of rotating turbulence with external forcing. Phys. Fluids 10, 2895-2909.

YoON, K. \& WARHAFT, Z. 1990 The evolution of grid-generated turbulence under conditions of stable thermal stratification. J. Fluid Mech. 215, 601-638. 